\title{
Plastic anisotropy of $\gamma$-TiAl revealed by axisymmetric indentation
}

\author{
C. Zambaldi ${ }^{\mathrm{a}, *}$, D. Raabe ${ }^{\mathrm{a}}$ \\ ${ }^{a}$ Max-Planck-Institut für Eisenforschung, Max-Planck-Str. 1, Düsseldorf, Germany
}

\begin{abstract}
Single crystals of $\gamma$-TiAl cannot be grown in the near-stoichiometric compositions that are present inside two-phase $\gamma / \alpha_{2}$-microstructures with attractive mechanical properties. Therefore, the single crystal constitutive behavior of $\gamma$-TiAl was studied by nanoindentation experiments in single phase regions of these $\gamma / \alpha_{2}$-microstructures. The experiments were characterized by orientation microscopy and atomic force microscopy to quantify the orientation dependent mechanical response during nanoindentation. Further, they were analyzed by a 3D crystal plasticity finite element model that incorporated the deformation behavior of $\gamma$-TiAl. The spatially resolved activation of competing deformation mechanisms during indentation was used to assess their relative strengths. A convention was defined to unambiguously relate any indentation axis to a crystallographic orientation. Experiments and simulations were combined to study the orientation dependent surface pile-up. The characteristic pile-up topographies were simulated throughout the unit triangle of $\gamma$-TiAl and represented graphically in the newly introduced inverse pole figure of pile-up patterns. Through this approach, easy activation of ordinary dislocation glide in stoichiometric $\gamma$-TiAl was confirmed independently from dislocation observation by transmission electron microscopy.
\end{abstract}

Key words: Instrumented indentation, EBSD, AFM, crystal plasticity, AuCu prototype

\section{Introduction}

Intermetallic $\gamma$-TiAl based alloys are prospective high-temperature structural materials due to their low density of about $4 \mathrm{~g} / \mathrm{cm}^{3}$ and good creep and oxidation properties [1-5]. However, the room-temperature ductility of this material is low. The alloys exhibit pronounced plastic anisotropy on the microstructural length scale and better understanding of the intrinsic mechanical properties is needed for progress in alloy design.

Mecking et al. [6] pointed out a limitation of current knowledge about the micro-mechanics of $\gamma$-TiAl. Although the types of dislocations occurring in $\gamma$-TiAl based alloys have been extensively characterized by transmission electron microscopy (TEM), e. g. [7-15], their densities and slip distances are not known and, therefore, the contributions of different kinds of dislocations to the overall deformation remain to be determined.

Better knowledge of the intrinsic single phase mechanical properties of the constituent phases could greatly enhance our understanding of the mechanically relevant microstructure-property relationships in $\gamma$-TiAl based alloys. Also, the strengthening contribution of the interfaces could be separated from the intrinsic properties.

\subsection{Nanoindentation and piling-up of material}

Since more than one-hundred years, indentation testing is an efficient and useful tool for mechanical characterization of materials and it is connected to the names of Brinell, Rockwell and others [16]. Progress in instrument design made possible the reduction of the indentation size and low load techniques were introduced [17-19]. Development of the instrumented indentation technique [20-23] lead to renewed interest in the fundamental aspects of indentation. Currently, simultaneous collection of load and displacement data can be routinely performed at resolutions on the order of nano-Newtons and fractions of a nanometer, respectively [24].

The literature on incipient plasticity during nanoindentation is omitted here because of space limitations. For aspects of atomistic modeling of dislocation nucleation and recent experimental findings on the initiation of plasticity during indentation, the reader is directed to [25-27].

\footnotetext{
${ }^{*}$ Corresponding author

Email address: c.zambaldi@mpie.de (C. Zambaldi)
} 


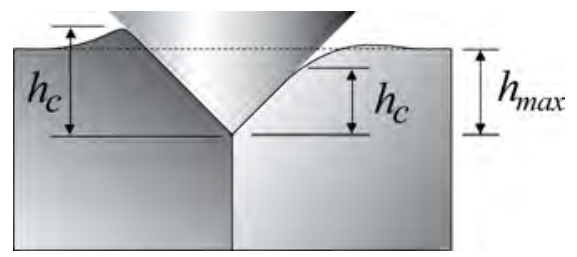

Figure 1: Material pile-up (left) and sink-in (right) increases or decreases the contact height, $h_{c}$, during indentation to the maximum penetration depth, $h_{\max }$.

Brookes et al. [28] presented the anisotropic hardness response of a number of single crystals. Indentations where performed with Knoop tools which themselves exhibit a strong geometrical anisotropy by an approximate length to width ratio of $7: 1$. Therefore, the results reflected the interaction between the plastic anisotropy of the crystal and the shape anisotropy of the indenter.

For the indentation of crystals, the topography of the free surface around the indent, the pile-up profile, can be used as a finger print of the underlying crystal deformation processes. In the 1920s, Tammann and Müller [29] have reviewed the characteristic patterns evolving around indents made with gramophone or sewing needles. They focused on indentation into high-symmetry planes of copper, $\alpha$-iron, and $\gamma$-iron, as well as the basal plane of zinc. The experimental findings on iron were taken from the pioneering work of Osmond and Cartaud [30, 31]. Already Osmond and Cartaud observed that the deformation patterns around a conical indent in single crystals is characteristic of the structure of the indented crystal, as well as for the orientation of the indented plane. More recently, the atomic force microscopy (AFM) technique enabled precise characterization of pile-up topographies and facilitated the study of deformation mechanisms during indentation $[32,33]$.

The shape and amount of anisotropic indentation pile-up in crystalline materials is depending on the indented orientation. It forms a source of uncertainty in applying the standard methods for the calculation of hardness and elastic properties [34]. Figure 1 illustrates how piling-up or sinking-in of material around the indenter can change the actual contact area between tool and sample. Correspondingly, Fischer-Cripps [35] stated: "Piling-up is perhaps the most important unresolved issue in nanoindentation testing".

Originally, indentation studies were often based on the assumption of isotropic elasto-plastic properties of the indented material. The finite element method has promoted understanding of indentation [36-45]. Mostly two-dimensional or axisymmetric models were applied. In indentation experiments at low loads, single crystal indentation prevails as the crystallites are often of comparable or larger size than the indents. In this case, the use of the crystal plasticity finite element method (CP-FEM) makes it possible to account for the deformation on discrete glide systems. It enables a realistic description of the anisotropic material flow during indentation. CPFEM simulation of indentation has been applied to copper [46-51], minerals [52], $\beta$-titanium [53], zinc [51], and a single-crystal superalloy [54].

\subsection{Crystal plasticity and indentation testing of $\gamma$-TiAl}

Gamma-TiAl has a fcc-derived tetragonal structure. It is $\mathrm{L} 1_{0}$-ordered with every second $\left(\begin{array}{lll}0 & 0 & 2\end{array}\right)$ plane containing only $\mathrm{Ti}$ or $\mathrm{Al}$ atoms. The unit cell exhibits a small tetragonality with a $c / a$ ratio of about 1.02 . The slip systems of $\gamma$-TiAl are similar to the twelve octahedral slip systems of the fcc structure, table 1 . However, glide of ordinary dislocations with a $c$-component would destroy the intermetallic ordering structure and therefore superdislocations with a Burgers vector of $\boldsymbol{b}^{\mathrm{S}}=\left[\begin{array}{lll}1 & 0 & 1\end{array}\right]$ are observed along eight of the twelve fcc shear directions. The remaining four slip directions show the usual Burgers vector of $\boldsymbol{b}^{\mathrm{O}}=1 / 2\left[\begin{array}{lll}1 & 1 & 0\end{array}\right]$.

Superdislocations with Burgers vector $1 / 2\left[\begin{array}{lll}1 & 1 & 2\end{array}\right]$ were reported to exist in $\gamma$-TiAl after room temperature deformation $[10,55]$. They are not taken into account in the following because they are expected to form sessile configurations at room temperature and will presumably only contribute to plastic deformation at elevated temperatures $[3,56]$.

Additionally, four true twinning systems, one per $\left\{\begin{array}{lll}1 & 1 & 1\end{array}\right\}$-plane, with Burgers vector $\boldsymbol{b}^{\mathrm{T}}=1 / 6\left[\begin{array}{lll}1 & 1 & \overline{2}\end{array}\right]$ can be activated $[57,58]$. True twinning refers to the four twinning directions in $\gamma$-TiAl, which do not generate an order fault at the twin interface.

Two-phase $\gamma / \alpha_{2}$-microstructures are promising for high-temperature structural applications. Most common are lamellar microstructures which contain minor fractions of the hexagonal, $\mathrm{D}_{19}$-ordered $\alpha_{2}-\mathrm{Ti}_{3} \mathrm{Al}$ phase. The 
Table 1: Deformation systems in $\gamma$-TiAl; Four ordinary dislocation glide systems, Burgers vector $\boldsymbol{b}^{\mathrm{O}}$, lead to shear without a $c$-component; Eight superdislocation systems, $\boldsymbol{b}^{\mathrm{S}}$, and four twinning systems, $\boldsymbol{b}^{\mathrm{T}}$, can provide shear deformation with a $c$-component.

\begin{tabular}{|c|c|c|c|c|c|c|c|c|}
\hline & & $\left(\begin{array}{lll}\overline{1} & \overline{1} & 1\end{array}\right)$ & & $(1 \overline{1} \overline{1})$ & & $\left(\begin{array}{lll}\overline{1} & 1 & \overline{1}\end{array}\right)$ & & $\left(\begin{array}{lll}1 & 1 & 1\end{array}\right)$ \\
\hline $\boldsymbol{b}^{S}$ & no. 1 & {$\left[\begin{array}{lll}0 & 1 & 1\end{array}\right]$} & no. 4 & {$\left[\begin{array}{lll}0 & \overline{1} & 1\end{array}\right]$} & no. 7 & {$\left[\begin{array}{lll}0 & 1 & 1\end{array}\right]$} & no. 10 & {$\left[\begin{array}{lll}0 & 1 & \overline{1}\end{array}\right]$} \\
\hline $\boldsymbol{b}^{S}$ & no. 2 & {$\left[\begin{array}{lll}1 & 0 & 1\end{array}\right]$} & no. 5 & {$\left[\begin{array}{lll}1 & 0 & 1\end{array}\right]$} & no. 8 & {$\left[\begin{array}{lll}1 & 0 & 1\end{array}\right]$} & no. 11 & {$\left[\begin{array}{lll}1 & 0 & 1\end{array}\right]$} \\
\hline $\boldsymbol{b}^{O}$ & no. 3 & $1 / 2\left[\begin{array}{lll}1 & 1 & 0\end{array}\right]$ & no. 6 & $1 / 2\left[\begin{array}{lll}\overline{1} & 1 & 0\end{array}\right]$ & no. 9 & $1 / 2\left[\begin{array}{lll}1 & 1 & 0\end{array}\right]$ & no. 12 & $1 / 2\left[\begin{array}{lll}1 & 1 & 0\end{array}\right]$ \\
\hline $\boldsymbol{b}^{T}$ & no. 13 & $1 / 6\left[\begin{array}{lll}1 & 1 & 2\end{array}\right]$ & no. 14 & $1 / 6\left[\begin{array}{lll}\overline{1} & 1 & \overline{2}\end{array}\right]$ & no. 15 & $1 / 6\left[\begin{array}{lll}1 & \overline{1} & \overline{2}\end{array}\right]$ & no. 16 & $1 / 6\left[\begin{array}{lll}1 & 1 & 2\end{array}\right]$ \\
\hline
\end{tabular}

$\gamma$-phase is formed from $\alpha_{2}$-phase, or its disordered high-temperature equivalent, during cooling [59]. Inside these microstructures the $\gamma$-phase contains approximately 49 at.\% Al [59]. It is difficult to obtain single crystal $\gamma$-TiAl of this near-stoichiometric composition [60]. Bulk single crystals of stoichiometric and Ti-rich $\gamma$-TiAl cannot be grown. Therefore, single crystal experiments on the plastic deformation of $\gamma$-TiAl could only be carried out for Alrich compositions, but for the stoichiometric $\gamma$-phase the experiments were restricted to the analysis of deformed polycrystalline material, mostly by TEM.

To overcome this limitation, Mahapatra et al. [61] produced a $150 \mu \mathrm{m}$ thick layer of near-stoichiometric $\gamma$-TiAl at the interface of a diffusion couple between Ti-56Al and Ti-39Al (at.\%) after annealing at $1265^{\circ} \mathrm{C}$ for 144 hours. This layer has then been co-deformed in compression with the matrix. From their observations, Mahapatra et al. [61] concluded that twinning is the primary deformation mechanism in stoichiometric $\gamma$-TiAl. This general conclusion is not supported by the results of the present work as will be discussed later.

Gamma-TiAl exhibits a remarkable dependence of the relative strength of the deformation systems on Alcontent [62]. For near-stoichiometric and Al-lean compositions, TEM analysis has revealed activation of ordinary dislocations and twinning at room temperature [55]. In contrast, for over-stoichiometric Al-contents, mainly deformation by superdislocations was observed [63, 64].

It should be noted that besides the effect of stoichiometry, the relative activities of ordinary and superdislocations depend also on the level of interstitial impurities. The influence of elements such as oxygen or nitrogen has been studied by several authors for the case of single $\gamma$-phase Ti-52Al [65-68]. For this composition, ordinary dislocations dominated the deformation microstructures for low impurity levels, whereas superdislocations prevailed in alloys containing higher amounts of interstitials. In the case of two-phase microstructures, the $\alpha_{2}$-phase has a higher solubility for oxygen in comparison to $\gamma$-TiAl and possibly reduces the oxygen content of the $\gamma$-phase [7, 69]. Indeed, recent results by Morris [70] suggest that, also in two-phase alloys, the oxygen level in the $\gamma$-phase constitutes an important parameter for the mobility of $1 / 2\left[\begin{array}{lll}1 & 1 & 0\end{array}\right]$ dislocations. However, since the mechanisms of the effect of Al-content and interstitial elements on the relative dislocation activities are not fully established, the term 'near-stoichiometric' will be used in the following, having in mind the possible influence of the oxygen concentration.

Nanoindentation of Ti-Al alloys has previously been studied. Göken et al. [71] observed anisotropic pile-up behavior around Berkovich indentations in $\gamma$-TiAl and $\alpha_{2}-\mathrm{Ti}_{3} \mathrm{Al}$. For the analysis of plastic anisotropy, Kempf et al. [72] recommended axisymmetric indenter shapes because they do not interfere with the plastic anisotropy of the crystal under consideration. Zhao et al. [73] performed cube corner indentations in lamellar material and reported the indentation size effect in $\gamma$-TiAl to be the lowest when compared to the following materials: $\mathrm{Ag}>\mathrm{Ni}$ $>\mathrm{Al}>\mathrm{Cu}>\alpha_{2}-\mathrm{Ti}_{3} \mathrm{Al}>\gamma$-TiAl. The internal length scale after Nix and Gao [74] is therefore smaller in the two Ti-Al phases than in pure metals with fcc structure. For the cube corner geometry, the hardness values approached size independence above indentation depths of about $250 \mathrm{~nm}$.

In this paper the application of small-scale indentation to the intermetallic phase $\gamma$-TiAl is presented. GammaTiAl has a complex deformation behavior and the capability of CP-FEM modeling to improve the understanding of the indentation process is demonstrated. The core contribution of this study is the characterization of the anisotropic plasticity of $\gamma$-TiAl, obtained through careful analysis of the pile-up behavior in combination with 3D CP-FEM modeling. The layout of the article is as follows: After describing the experimental and computational methods, results of nanoindentation experiments in known orientations of $\gamma$-TiAl are presented. Then, a theoretical framework consisting of an orientation convention and a discretization of orientation space is introduced for the presentation of the data. The convention is used to compare experimental and computational results on the orientation dependent deformation of the free surface around the indents. Specific orientations are explored in detail, to investigate and explain the formation mechanisms of indentation pile-up in the case of $\gamma$-TiAl. Altogether, the presented techniques form a novel systematic approach to the analysis of piling-up during single crystal indentation 
which constitutes a new tool to quantify plastic anisotropy.

\section{Experimental procedures}

Cylindrical samples ( $\mathrm{D}=6 \mathrm{~mm}$ ) of Ti-45.9Al-8Nb (at.\%), grown in an optical floating zone furnace [75] at a withdrawal rate of $10 \mathrm{~mm} / \mathrm{h}$, were provided by IFW-Dresden. Discs of $4 \mathrm{~mm}$ thickness were cut by wire electrical discharge machining, ground to 1000 grit, polished with $3 \mu \mathrm{m}$ diamond suspension, and finally electrolytically polished at $-30^{\circ} \mathrm{C} / 35 \mathrm{~V}$ in a solution of $6 \%$ perchloric acid in ethanol. The microstructure, as observed by backscatter electron (BSE) imaging, was almost fully lamellar, with a lamellar colony size of few hundred micrometers and small $\gamma$-TiAl grains along part of the grain boundaries. The crystallographic orientation of the $\gamma$-phase was characterized by the electron backscatter diffraction (EBSD) technique. The correct indexing of the $\gamma$-TiAl order variants was based on high accuracy measurement parameters and a recently developed indexing procedure [76].

A Hysitron TriboScope 900 was used for indentation. The maximum applied load was $10 \mathrm{mN}$. Instrumented indentation was performed with indenters of Berkovich and sphero-conical tip geometries. Generally, the lamellar structure was too fine to perform sphero-conical indentation inside the lamellae. Indentations were mainly made in a region of coarser $\gamma$-grains of about $20 \mu \mathrm{m}$ grain size. Some indents were also placed in coarse $\gamma$-phase lamellae. The remaining indent topographies were analyzed by a Veeco dimension 3100 atomic force microscope (AFM).

\section{Simulation of indentation by the crystal plasticity finite element method}

\subsection{Incorporating the $\gamma$-TiAl deformation mechanisms into an elasto-viscoplastic crystal plasticity model}

An existing crystal plasticity model [77, 78], implemented after the works of Kalidindi et al. [79], was adapted to the characteristics of $\gamma$-TiAl. A short summary is given in the following: the kinematic description of large-strain elasto-plasticity is based on the multiplicative decomposition of the deformation gradient, $\boldsymbol{F}$, as $\boldsymbol{F}=\boldsymbol{F}^{*} \boldsymbol{F}^{p}$, into the plastic part, $\boldsymbol{F}^{p}$, and $\boldsymbol{F}^{*}$, containing elastic stretch and rotation [80]. The plastic velocity gradient, $\boldsymbol{L}^{p}$, is used in the formulation of the constitutive model and can be calculated from $\boldsymbol{L}^{p}=\dot{\boldsymbol{F}}^{p} \boldsymbol{F}^{p-1}$. Deformation of crystals by dislocation glide can be described by the Orowan equation, $\dot{\gamma}=b \rho_{\text {mobile }} v$, with the crystallographic shear rate, $\dot{\gamma}$, the Burgers vector length, $b$, the mobile dislocation density, $\rho_{\text {mobile }}$, and the dislocation glide velocity, $v$. However, the dislocation densities and velocities in $\gamma$-TiAl are not known. In this case, to be able to simulate the constitutive response, it is useful to retreat to the quantitative description of the shear rates resulting from the different types of dislocations.

Since only shear on the deformation systems is allowed to contribute to plastic deformation, $\boldsymbol{L}^{p}$ is calculated from the sum of the shear rates, $\dot{\gamma}^{\alpha}$, on all deformation systems, $\alpha$, as

$$
\boldsymbol{L}^{p}=\dot{\boldsymbol{F}}^{p} \boldsymbol{F}^{p-1}=\sum_{\alpha} \dot{\gamma}^{\alpha} \boldsymbol{d}_{0}^{\alpha} \otimes \boldsymbol{n}_{0}^{\alpha} .
$$

The unit vectors $\boldsymbol{d}_{0}^{\alpha}$ and $\boldsymbol{n}_{0}^{\alpha}$ indicate the shear direction and the normal on the glide plane of the slip system $\alpha$, respectively. The index zero is given since in this total Lagrangian formulation the slip plane normals and slip directions are assumed to be constant throughout the calculation [81]. Shear rates, $\dot{\gamma}^{\alpha}$, are taken as a visco-plastic function of the resolved shear stresses, $\tau^{\alpha}$, on the deformation systems [82, 83]

$$
\dot{\gamma}^{\alpha}=\dot{\gamma}_{0}\left|\frac{\tau^{\alpha}}{\tau_{c}^{\alpha}}\right|^{1 / m} \operatorname{sign}\left(\tau^{\alpha}\right)
$$

where $\dot{\gamma}_{0}$ and $m$ are material parameters.

The slip resistances, $\tau_{c}^{\alpha}$, evolve with the shear rates, $\dot{\gamma}^{\beta}$, of all systems (index $\beta$ ) according to

$$
\dot{\tau}_{c}^{\alpha}=\sum_{\beta} h^{\alpha \beta}\left|\dot{\gamma}^{\beta}\right|, \quad h^{\alpha \beta}=q^{\alpha \beta} h^{(\beta)},
$$

with the "instantaneous moduli" [84], $h^{\alpha \beta}$, also referred to as hardening matrix. It is calculated from the selfhardening, $h^{(\beta)}$, and the cross-hardening matrix, $q^{\alpha \beta}$, with its diagonal elements equal to unity. Typically, in fcc 
crystals, the values of $q^{\alpha \beta}$ are chosen to be 1.4 for non-coplanar and 1 for coplanar slip [85]. The hardening contribution from self-hardening, $h^{(\beta)}$, is given by:

$$
h^{(\beta)}=h_{0}^{\beta}\left(1-\frac{\tau^{\beta}}{\tau_{s}^{\beta}}\right)^{a^{\beta}}
$$

Note the change in comparison to the original formulation [79] from constant values, $h_{0}, \tau_{s}, a$, for all slip systems, to $h_{0}^{\beta}, \tau_{s}^{\beta}, a^{\beta}$, now being dependent on the individual characteristics of the slip systems.

This leaves four parameters for the description of the flow behavior: the initial slip resistance, $\tau_{0}^{\beta}$; the saturation slip resistance, $\tau_{s}^{\beta}$; the parameter $h_{0}^{\beta}$ which determines the initial hardening slope; and the hardening exponent, $a^{\beta}$, which influences the shape of the self-hardening curve.

Since the primary meaning of the reference shear rate, $\dot{\gamma}_{0}$, and the strain rate sensitivity parameter, $m$, in the scope of the present work is rather of a numerical nature than being related to the micro-mechanical properties, they are assumed constant in all simulations. The parameter $m$ is suitable to include strain rate sensitivity of the flow stress into the model. To approximate time-independent behavior its value is kept small. However, too small values make robust convergence of the time-integration procedure difficult.

Time-integration of the constitutive law is achieved through the implicit scheme by Kalidindi et al. [79]. Since different values of $\tau_{0}$ are allowed for, convergence is tested against the minimum value of all $\tau_{0}^{\alpha}$. The tolerance for the inner stress integration loop is chosen to be $10^{-4} \times \min \left(\tau_{0}^{\alpha}\right)$. The outer loop reaches convergence when the maximum change in the components of the calculated shear strengths becomes smaller than the minimum initial shear strength of all systems, $\max \left(\Delta \tau_{c}^{\alpha}\right)<10^{-3} \times \min \left(\tau_{0}^{\alpha}\right)$.

The incorporation of twinning systems into crystal plasticity formulations has been discussed by Kalidindi [86]. In this work four $\left\{\begin{array}{lllll}1 & 1 & 1\end{array}\right\}\left[\begin{array}{lll}1 & 1 & \overline{2}\end{array}\right]$ true twinning systems (table 1$)$ were implemented as uni-directional slip systems: if the resolved shear stress on the twinning plane is positive, the stress integration is carried out in the same manner as for the dislocation glide systems; if the resolved shear stress is negative, the shear on this glide system will be set to zero for the current increment.

In reality, twinning leads to reorientation of the twinned material volume. This reorientation is neglegted in the present model. Further, twinning in $\gamma$-TiAl cannot produce shear values higher than $\left(2(c / a)^{2}-1\right) /(c / a \sqrt{2}) \approx 0.75$ [87], with a tetragonal $c / a$ ratio of 1.02 , corresponding to a shear angle of approximately $37^{\circ}$. This limitation is not incorporated in the model since the maximum shear value for a twinned volume fraction of 1 is relatively high and appropriate hardening behavior makes it virtually impossible to reach this value in significant volumes.

\subsection{Finite element simulations}

The crystal plasticity formulation was integrated into the finite element method (FEM) by using the material subroutine hypela2 of the commercial FEM system MARC [88]. A three-dimensional FE-model of the indentation process was generated. Deformation of the sphero-conical indenter was neglected by assuming a rigid body. The sample was discretized by 4320 hexahedral 8 node elements. Solutions at this level of discretization were consistent with simulations using finer meshes, figure 2 .

The exact tip geometry was not known a priori. Therefore, an initial series of simulations was performed, to adjust the tip radius. Iteratively, the tip radius of the simulations was changed until the difference between the simulated and the measured remaining indent topographies became minimal.

Friction is known to have a minor effect on the load-displacement response of indentation [36]. However, Liu et al. [49] have shown that the value of the coefficient of friction might affect the maximum height of the formed pile-up. Simulations with the present model confirmed that the maximum pile-up height decreases by about $30 \%$ ([5 $\left.\begin{array}{ll}0 & 0\end{array}\right]$ indentation) for increasing friction coefficients of $0.1,0.2,0.3$ and 0.4 and afterwards remains virtually constant up to the highest simulated value of 1.0. A coefficient of friction of 0.3 was assumed in the simulations. Friction was found to reduce the mesh distortion close to the contact between indenter and sample.

The elastic constants $c_{11}=182.8 \mathrm{GPa}, c_{33}=176.9 \mathrm{GPa}, c_{12}=75.2 \mathrm{GPa}, c_{44}=103.5 \mathrm{GPa}, c_{66}=76.5 \mathrm{GPa}$, measured on mono-crystalline Ti-56Al (at.\%), were used [89]. Different material parameters for the simulation of inelastic deformation were assigned for each of the three types of deformation systems: table 2 presents the values for the self-hardening parameters. The strain rate sensitivity parameter, $m$, was set to 20 and the reference shear rate, $\dot{\gamma}_{0}$, was $0.001 \mathrm{~s}^{-1}$. 


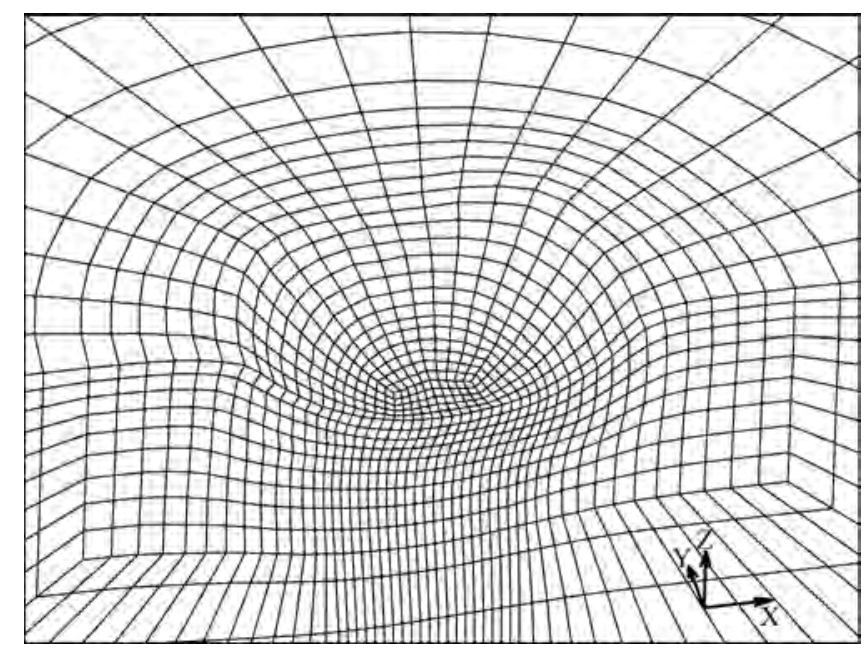

Figure 2: A finite element mesh of 15060 hexahedral elements after indentation; this was the maximum resolution of the indentation process, which was used for validation of the results with coarser meshes. One half on the model is not shown for better visibility of the mesh structure.

Table 2: Parameters of the crystal plasticity model for ordinary dislocation glide $\left(\boldsymbol{b}^{\mathrm{O}}\right)$, superdislocation glide $\left(\boldsymbol{b}^{\mathrm{S}}\right)$ and twinning $\left(\boldsymbol{b}^{\mathrm{T}}\right)$ systems from table $1, m=20, \dot{\gamma}_{0}=0.001 \mathrm{~s}^{-1} ; \tau_{0}$ is the initial slip resistance, $\tau_{s}$ the saturation slip resistance, $h_{0}$ influences the initial hardening slope and $a$ is the hardening exponent, equation (4);

\begin{tabular}{ccccc}
\hline & $\tau_{0}, \mathrm{MPa}$ & $\tau_{s}, \mathrm{MPa}$ & $h_{0}, \mathrm{MPa}$ & $a$ \\
\hline $\boldsymbol{b}^{\mathrm{O}}$ & 55 & 400 & 300 & 2.5 \\
$\boldsymbol{b}^{\mathrm{S}}$ & 165 & 1200 & 300 & 2.5 \\
$\boldsymbol{b}^{\mathrm{T}}$ & 110 & 800 & 300 & 2.5 \\
\hline
\end{tabular}

For the cross-hardening parameters, $q^{\alpha \beta}$, the values 1 and 1.4 were chosen for co-planar and non-co-planar deformation systems, respectively. These values can only be a fair estimate and might deviate from the true crosshardening interactions. However, because of the impossibility of bulk single crystal experiments on stoichiometric $\gamma$-TiAl, no experimental data was reported up to now, which could substantiate the use of different values.

\section{Results}

\subsection{Experimental results}

Several crystallographic orientations of $\gamma$-TiAl were indented inside the two-phase microstructure. Figure 3 shows a region of the nearly lamellar microstructure with some $\gamma$-TiAl grains. A square grid of Berkovich indents, made with a maximum load of $3 \mathrm{mN}$, is also visible. After Berkovich indentation, the area was characterized by EBSD orientation mapping. Additional indents with axisymmetric indenters were then placed between the Berkovich indents. Load-displacement data for axisymmetric indentation of a specific crystal orientation is given in figure 4. Indentation axes of some indented $\gamma$-grains are listed in table 3 together with the respective maximum indentation depths.

Figure 5 gives a combined view of AFM data overlayed with an orientation map, colored by the EBSD inverse pole figure coloring scheme. Glide steps were observed to be in agreement with slip on closest packed $\left\{\begin{array}{lll}1 & 1 & 1\end{array}\right\}$ planes, however, it is not clear whether these surface steps are related to dislocation glide or twinning activity.

Based on the observation of pile-up in several orientations, an underlying principle of the pile-up shape was deduced. One-dimensional properties such as the uniaxial stiffness are identical for two crystal directions that are 

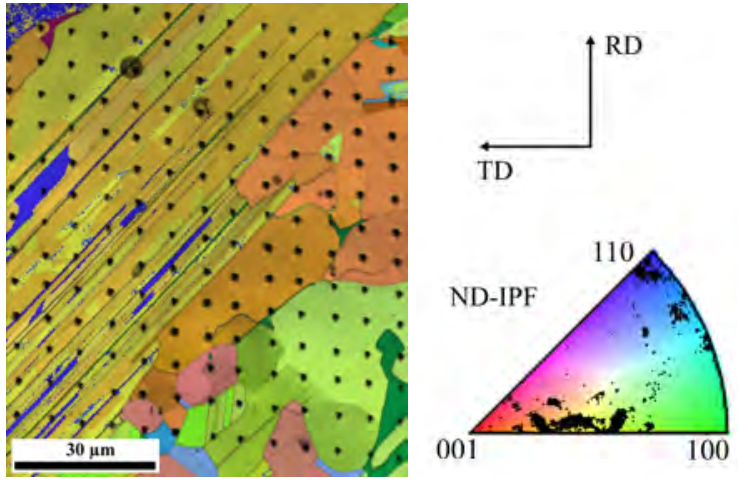

Figure 3: A region with $\gamma$-grains in the nearly lamellar microstructure; EBSD orientation map acquired with the recently developed fit-rank indexing. The crystallographic orientation in color is overlaid with the image quality measure in greyscale. Berkovich indents, made with a maximum load of $3 \mathrm{mN}$ and arranged on a square grid with a nominal spacing of $6 \mu \mathrm{m}$, are visible through a reduced pattern quality. The inverse pole figure coloring scheme is also shown with the measured orientations in black. Indentations with an axisymmetric indenter were applied in selected regions of the microstructure.

Table 3: Experimentally measured orientations and maximum indentation depths for several indents with a maximum load of $10 \mathrm{mN}$;

\begin{tabular}{|c|c|}
\hline ind. axis $[u v w]$ & $h_{\max }, \mathrm{nm}$ \\
\hline 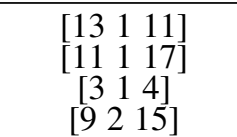 & $\begin{array}{c}345,347,316^{\mathrm{a}} \\
368,369,345,348 \\
334,338 \\
369,338^{\mathrm{a}}\end{array}$ \\
\hline$\left[\begin{array}{lll}14 & 13 & 2\end{array}\right]$ & $329^{\mathrm{b}}, 335^{\mathrm{b}}$ \\
\hline$\left[\begin{array}{lll}1 & 10 & 6\end{array}\right]$ & $311^{\mathrm{a}, \mathrm{b}}, 321^{\mathrm{b}}, 317^{\mathrm{b}}, 320^{\mathrm{b}}$ \\
\hline
\end{tabular}

a Probably influenced by grain boundaries;

${ }^{b}$ Different tip with a smaller tip radius and load $6 \mathrm{mN}$;

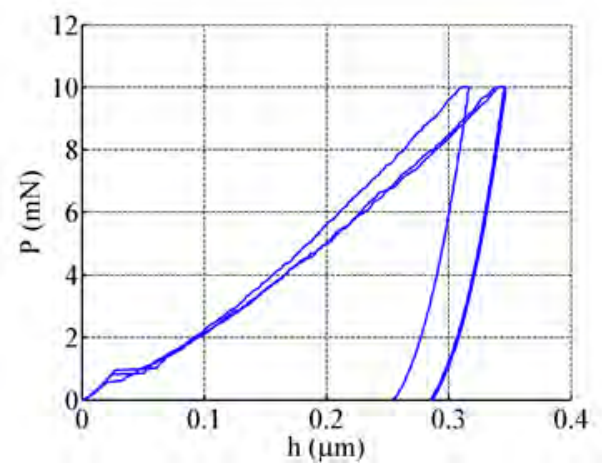

Figure 4: Load-dislacement curves for near-[ $\left.\begin{array}{lll}1 & 0 & 1\end{array}\right]$ indentation along [ $\left[\begin{array}{lll}3 & 1 & 11\end{array}\right]$. Loading and unloading rate was $2 \mathrm{mN} / \mathrm{s}$ with a dwell time of $1 \mathrm{~s}$; Initial Hertzian loading and subsequent pop-in is observed. One of the three curves was measured close to a grain boundary and higher hardening is observed in this case [90]. 

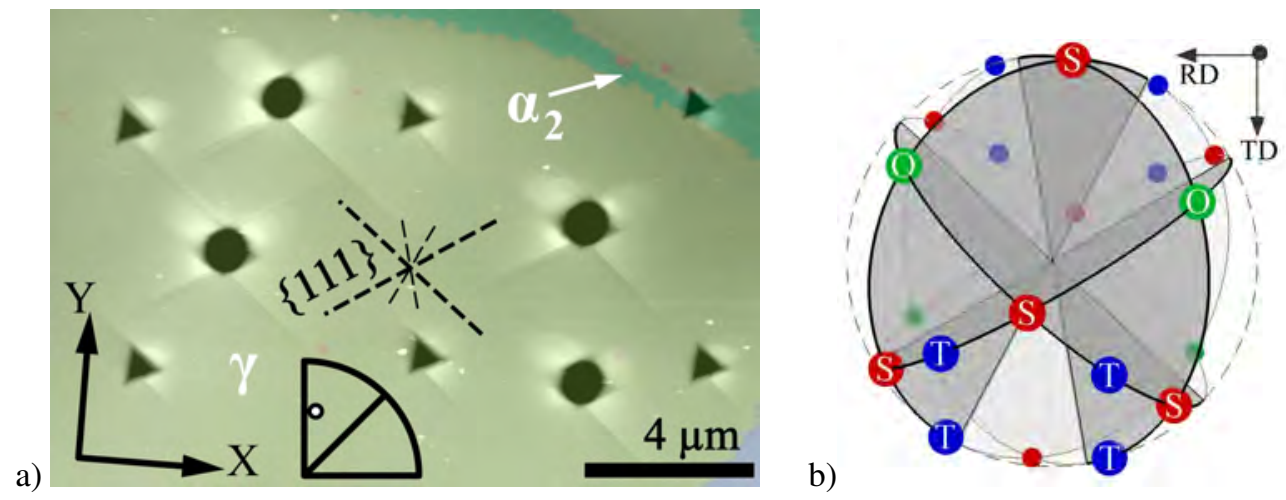

Figure 5: a) Nanoindents in a $\gamma$-grain of nearly lamellar Ti-46Al-8Nb (at.\%); AFM topographic map (greyscale) combined with an EBSD orientation map ([ $\left[\begin{array}{lll}0 & 0 & 1\end{array}\right]$ inverse pole figure coloring, c. f. figure 3). The $\left\{\begin{array}{lll}1 & 1 & 1\end{array}\right\}$-traces coincide with the observed surface steps. Sphero-conical (nominal tip radius $R<1 \mu \mathrm{m}$ ) and Berkovich indentations were performed with maximum loads of $6 \mathrm{mN}$ and $3 \mathrm{mN}$, respectively. Indentation axes were oriented along [ $\left[\begin{array}{lll}1 & 10 & 6\end{array}\right]$, the $X-Y$ coordinates are oriented after the convention that is defined in section 4.2 . b) Illustration of the crystal orientation of the $\gamma$-phase in subfigure (a) (perspective projection): the (llllllanes are shown together with the shear directions for ordinary dislocation glide $(\mathrm{O}$, green), superdislocation glide $(\mathrm{S}, \mathrm{red})$ and twinning ( $\mathrm{T}$, blue);

related through an improper symmetry operation ${ }^{1}$. The formation of indentation pile-up additionally involves the lateral dimensions and, therefore, the pile-up shape is not a uniaxial property of the crystal. Correspondingly, the pile-up shapes of two equivalent indentation axes that are related to each other by an improper symmetry operation, exhibit mirror symmetry, figure 6. It follows that, for the representation of experimental indentation axes, a standard orientation triangle should be used that can be generated by using only proper symmetry operations based on orthogonal matrices with determinant +1 . It is twice as large as the one that also allows for improper rotations based on orthogonal matrices with determinant -1 [91]. This result also holds for pile-up patterns in materials with other crystal structures.

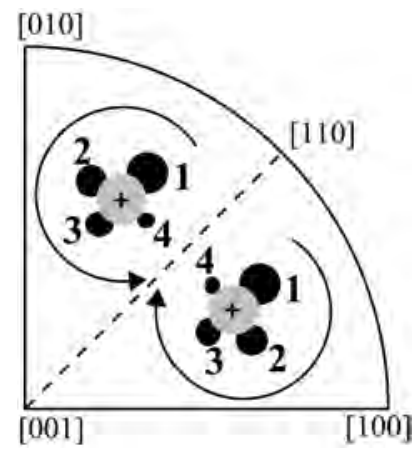

Figure 6: The pile-up topography is changed under influence of an improper symmetry operation; the developed surface profiles change their sense of rotation. The pile-up patterns of two indented orientations of $\gamma$-TiAl, connected by an improper symmetry operation, exhibit mirror symmetry through the $\left(\begin{array}{lll}1 & 1 & 0\end{array}\right)$ plane.

\subsection{A convention to display pile-up topographies in an unambigous manner}

For axisymmetric indenter geometries, an indentation axis $[u v w]$ leaves a rotational degree of freedom around this axis, i. e. there exists an infinite number of crystallographic orientations with the same indentation axis. To directly compare measured or simulated indent topographies, this rotational degree of freedom needs to be removed by defining a unique coordinate frame for every indentation axis.

A thought experiment is employed to define a convention for displaying pile-up topographies throughout the orientation triangle. Figure 7 illustrates the thought experiment, in which a spherical single crystal with a radius

\footnotetext{
${ }^{1}$ Symmetry operations are here represented by orthogonal matrices. An improper symmetry operation contains the inversion and changes the handedness of an object that it acts upon. In the case of $\gamma$-TiAl there are eight proper and eight corresponding improper symmetry operations.
} 

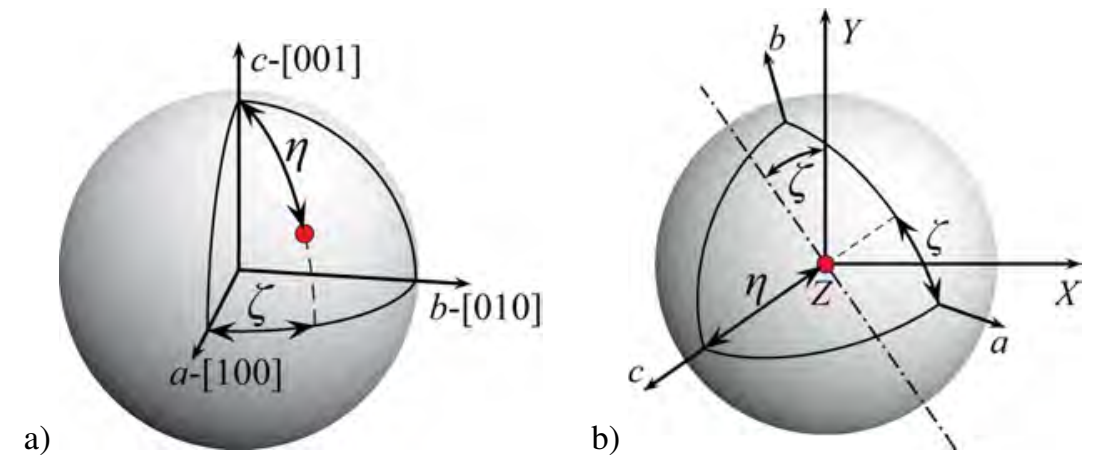

Figure 7: Illustration of the orientation convention for displaying pile-up topographies. a) The crystal coordinate system $\left[x_{c}, y_{c}, z_{c}\right]$ where $x_{c}\left\|a, y_{c}\right\| b, z_{c} \| c$; a desired indentation direction, defined by the spherical coordinates $(\zeta, \eta)=\left(35^{\circ}, 60^{\circ}\right)$, is marked by the red dot. b) The indenter coordinate system $[X, Y, Z]$ with the crystal rotated after the convention as defined in the text. $Z$ is anti-parallel to the indentation direction. The rotation axis is shown by the dot-dash line.

much larger than the indent dimensions is rotated under the indenter to reach any indentation axis on the surface. An orthonormal basis of the crystal is given by $\left[x_{c}, y_{c}, z_{c}\right]$. For $\gamma$-TiAl the crystal axes $a, b, c$ are taken parallel to these basis vectors, figure 7-a. The initial orientation is defined as $c$-axis $\| Z$ (indentation direction), $a$-axis $\| X$, $b$-axis $\| Y$, with the indenter coordinate system $[X, Y, Z]$. Starting from this reference orientation, an unambiguous crystallographic orientation can be defined, based on the spherical coordinates $(\zeta, \eta)$. First, a rotation axis is defined by rotating the negative $Y$-axis by an angle $\zeta$ about the $Z$-axis. Second, the crystal is rotated by an angle $\eta$ around this rotation axis. Thereby the desired indentation axis will be aligned with the $Z$-axis of the indenter setup, figure 7-b. In this way, the convention is defined so that the projection of the indenter tip onto the crystal's surface describes the shortest possible trajectory during the alignment.

The topography after indentation is then displayed in the $X-Y$ system of the indenter, viewed along the negative $Z$-direction. This can easily be achieved for CP-FEM simulations by choosing a crystal orientation according to the convention. For experiments, pile-up topographies of arbitrary in-plane orientation are measured. To comply with the convention, the in-plane misorientation of the experimentally measured crystal orientation from the unique orientation has to be calculated and the topography is then rotated into the unique orientation.

The two angles $\zeta$ and $\eta$, as defined above, are easily related to the axis-angle representation of crystal orientations. The Bunge Euler angles can be calculated from them as $\left(\varphi_{1}, \Phi, \varphi_{2}\right)=\left(270^{\circ}+\zeta, \eta, 90^{\circ}-\zeta\right)$. Application of this convention is not limited to the crystal structure presented here.

\subsection{Simulation of orientation dependent pile-up patterns in TiAl throughout the unit orientation triangle}

Helming et al. [92] suggested a near-equidistant discretization of orientation space. The first stage of their method was used to generate a number of 21 directions in the standard orientation triangle of the fcc structure at an approximate resolution of $9^{\circ}$. By appropriate symmetry operations, these directions were expanded to fill the $\left[\begin{array}{lll}0 & 0 & 1\end{array}\right]-\left[\begin{array}{lll}1 & 0 & 0\end{array}\right]-\left[\begin{array}{lll}1 & 1 & 0\end{array}\right]$ unit triangle of the tetragonal structure. Using the convention defined above, the directions were converted to full crystallographic orientations. Details of the involved calculations and a list of the resulting Bunge Euler angles are given in Appendix A.

Indentation was simulated for 51 orientations in the unit triangle of $\gamma$-TiAl. Figure 8 presents the resulting pile-up topographies in a graph that we will refer to as the pile-up inverse pole figure (pile-up IPF). The individual topographies are placed at the inverse pole figure representation of their respective indentation axes. The topographies are in-plane oriented after the convention defined in section 4.2 with $X \|\left[\begin{array}{lll}1 & 0 & 0\end{array}\right]$ and $Y \|\left[\begin{array}{lllll}0 & 1 & 0\end{array}\right]$. To-

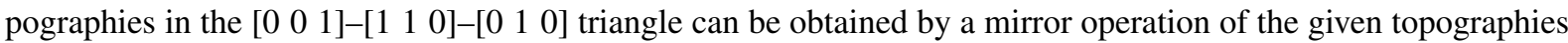
through the $\left(\begin{array}{lll}\overline{1} & 1 & 0\end{array}\right)$ plane, as described above, figure 6 . From the indentation simulations it was found that the chosen resolution of about $9^{\circ}$ provides sufficient detail throughout orientation space to enable smooth interpolation between the orientation points.

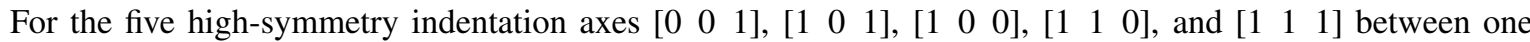
and four dominant hillocks were observed: for a $\left[\begin{array}{lll}0 & 0 & 1\end{array}\right]$ orientation axis a four-fold pile-up pattern, with four dominant hillocks in the $\left\langle\begin{array}{llll}1 & 1 & 0\end{array}\right\rangle$ directions, resulted from the simulation, similar to the pattern in fcc crystals. The $\left[\begin{array}{lll}1 & 0 & 1\end{array}\right]$ orientation exhibits two dominant hillocks in neighboring positions. A $\left[\begin{array}{lll}1 & 0 & 0\end{array}\right]$ indentation axis shows a 
pattern similar to the [llll $\left.\begin{array}{lll}0 & 0 & 1\end{array}\right]$ axis, but the four-fold symmetry is broken and only two-fold symmetry remains. The $\left[\begin{array}{lll}1 & 1 & 0\end{array}\right]$ indentation axis results in two major hillocks on opposite sides of the impression. For the case of $\left[\begin{array}{lll}1 & 1 & 1\end{array}\right]$-indentation only one dominant protuberance is formed.

The simulations showed that the phenomenology of the anisotropic pile-up topographies is mainly influenced not by the absolute, but by the relative strengths of the respective types of deformation systems. Additionally, the maximum pile-up height was found to be sensitive to the hardening behavior. Higher hardening resulted in shallower piling-up. This observation is in agreement with corresponding observations on poly-crystalline materials $[16,40]$.

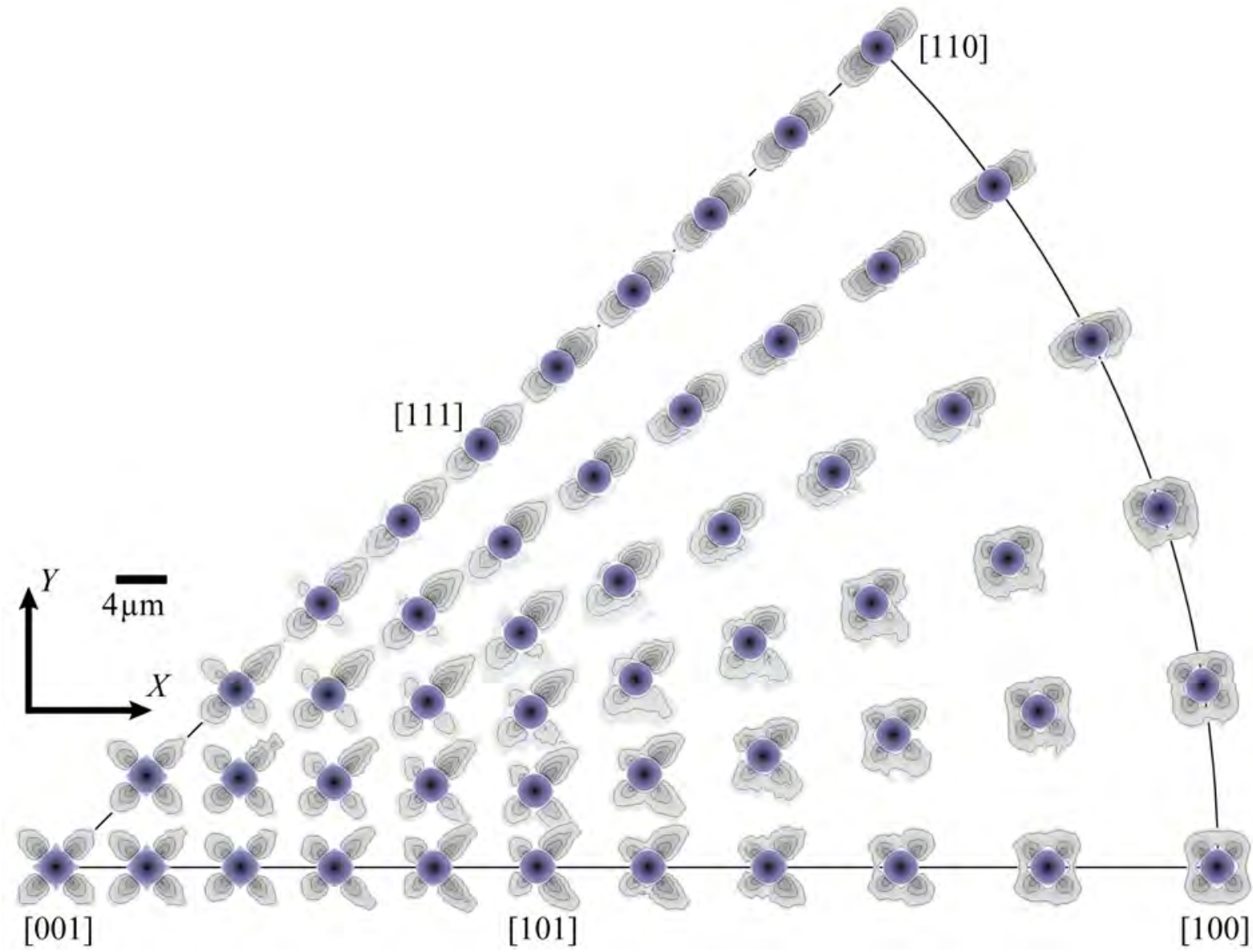

Figure 8: Predicted pile-up patterns from axisymmetric indentation in $\gamma$-TiAl. The topographic data were obtained from 51 CP-FEM simulations; sphero-conical tip with $146^{\circ}$ apex angle, tip radis $2.7 \mu \mathrm{m}$, maximum indentation depth $0.32 \mu \mathrm{m}$. The individual patterns are placed in an inverse pole figure (stereographic projection) of the respective indentation axes and in-plane oriented after the convention defined above, using the indicated indenter coordinates $X$ and $Y$. The impressions are shown in bluish color (contour lines at $-0.05,-0.1,-0.15 \mu \mathrm{m} \ldots$ ), the upheaval in greyscale (contour lines at 10, 20,30 nm ...). The number of dominant hillocks ranges from one, for a [ 1111$]$ indentation axis, to four, for axes $\left[\begin{array}{lll}0 & 0 & 1\end{array}\right]$ and $\left[\begin{array}{lll}1 & 0 & 0\end{array}\right]$.

\subsection{Comparison of experimental and simulated pile-up topographies}

Generally, experimental and simulated pile-up patterns showed good agreement. The observed pile-up shapes from figure 5 can be correlated to their respective counterparts in the predicted pile-up IPF, figure 8 , through a mirroring operation as described above. The further analysis will focus on indents made into orientations close to high-symmetry indentation axes.

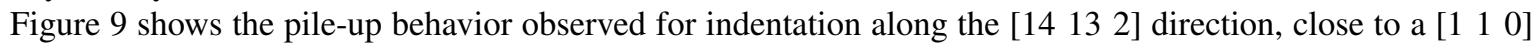
indentation axis. A sphero-conical indenter tip with a nominal tip radius smaller than $1 \mu \mathrm{m}$ was used to place the indents inside the coarse $\gamma$-phase lamella. Also, a reduced indentation load of $6 \mathrm{mN}$ was used to limit the size 
of the impressions. Two pile-up hillocks were formed on opposite sides of the impression. Very similar pile-up shapes are observed in the CP-FEM simulations of orientations near the $\left[\begin{array}{lll}1 & 1 & 0\end{array}\right]$ indentation axis, figure 8 . Due to the continuum character of the CP-FEM simulations, the observed slip traces will not occur in the finite-element results.
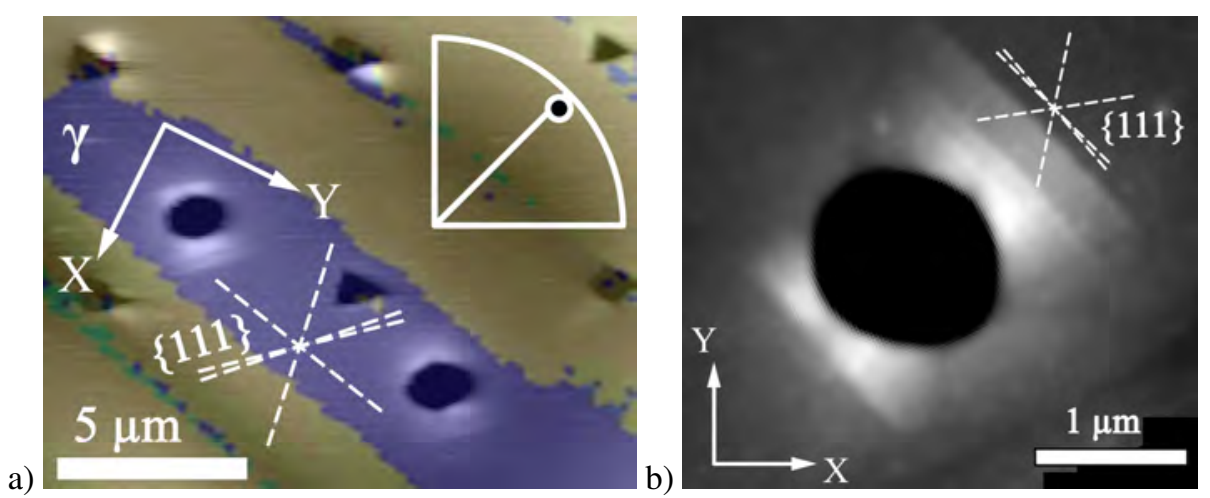

Figure 9: a) Topography (greyscale) of two sphero-conical indents in a coarse $\gamma$-phase lamella of orientation [14 13 2], c. f. the inverse polefigure projection of the indentation axis; color indicates grain orientations. The greyscale coloring ranges to about $18 \mathrm{~nm}$ surface elevation. The maximum indentation load was $6 \mathrm{mN}$. The inscribed $X-Y$ coordinate system is in-plane rotated after the defined convention. Four Berkovich indentations (maximum load $3 \mathrm{mN}$ ) are also visible. b) Higher resolution topography of one of these indents, in-plane rotated after the convention defined in section 4.2 .

Figure 10 compares an experimentally measured pile-up topography to the simulation result for a [ $\left.\begin{array}{lllll}1 & 0 & 1\end{array}\right]$ indentation axis. The pile-up topographies are displayed following the convention defined above. Agreement between the measured and simulated topographies is discussed below in terms of the activated deformation mechanisms.

\section{Discussion}

\subsection{Relation between surface deformation and plastic anisotropy}

The measurement of intrinsic single phase properties in complex alloys and compounds is of high importance. The mechanical response of real microstructures, such as two-phase $\gamma$-TiAl $/ \alpha_{2}-\mathrm{Ti}_{3} \mathrm{Al}$-microstructures, results from the combination of intrinsic properties with the influence of the interphase interfaces [93, 94]. Better understanding of these interface effects can only be gained through their separation from the single phase properties.

In the previous sections, a combined approach was presented to characterize and understand the plastic anisotropy of $\gamma$-TiAl during indentation. Orientation mapping of the indented crystallites was combined with the surface topographies from AFM measurements. CP-FEM simulations, based on the experimentally measured orientations and on the preexisting knowledge about possible deformation modes in $\gamma$-TiAl, then revealed the potential of our approach to link the orientation dependent pile-up patterns to the operating deformation systems. With the identified constitutive parameters, the orientation dependent pile-up behavior was predicted for all possible indentation axes to construct the pile-up IPF.

Height, shape and distribution of the emerging hillocks clearly reflect the distinct shear directions in the material on the individual deformation systems. These processes are observed at single phase level, thereby isolating the mechanical behavior from the influence of grain boundaries as well as lamellar and domain boundaries.

In principle, the indentation deformation could be influenced by the residual stresses in $\gamma$-TiAl based alloys that can result from the lattice mismatch between $\gamma$-domains and also at $\gamma / \alpha_{2}$-interfaces $[11,95,96]$. High misfit stresses have been estimated for lamellar microstructures with a coherent or semi-coherent interface structure [96]. For $\gamma$-grains that are surrounded by non-planar grain boundaries, smaller residual stresses are expected by the present authors. Further, a partial relaxation of the residual stresses at the free surface seems possible. In the $\mathrm{CP}$ FEM simulations, an initially stress free state was assumed. Because of the good agreement between the simulated and measured pile-up patterns, that was observed for all indents, the influence of residual stresses is assumed to be of minor relevance in the present examples.

The topography of the pile-up shape was a characteristic feature of the indented crystallographic direction as has been previously reported for indentation into high-symmetry orientations of copper [46]. In comparison 
a)
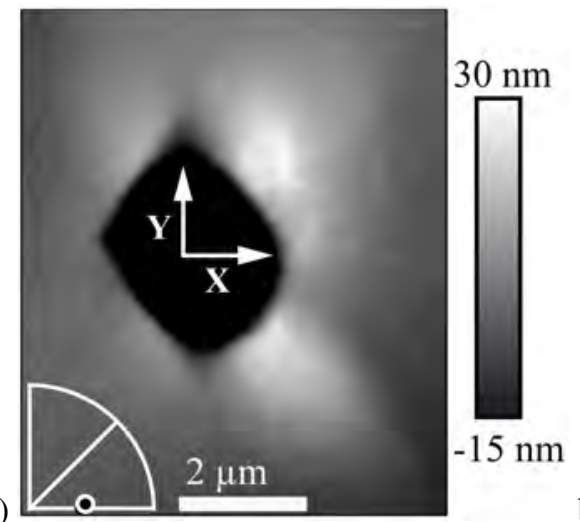

b)
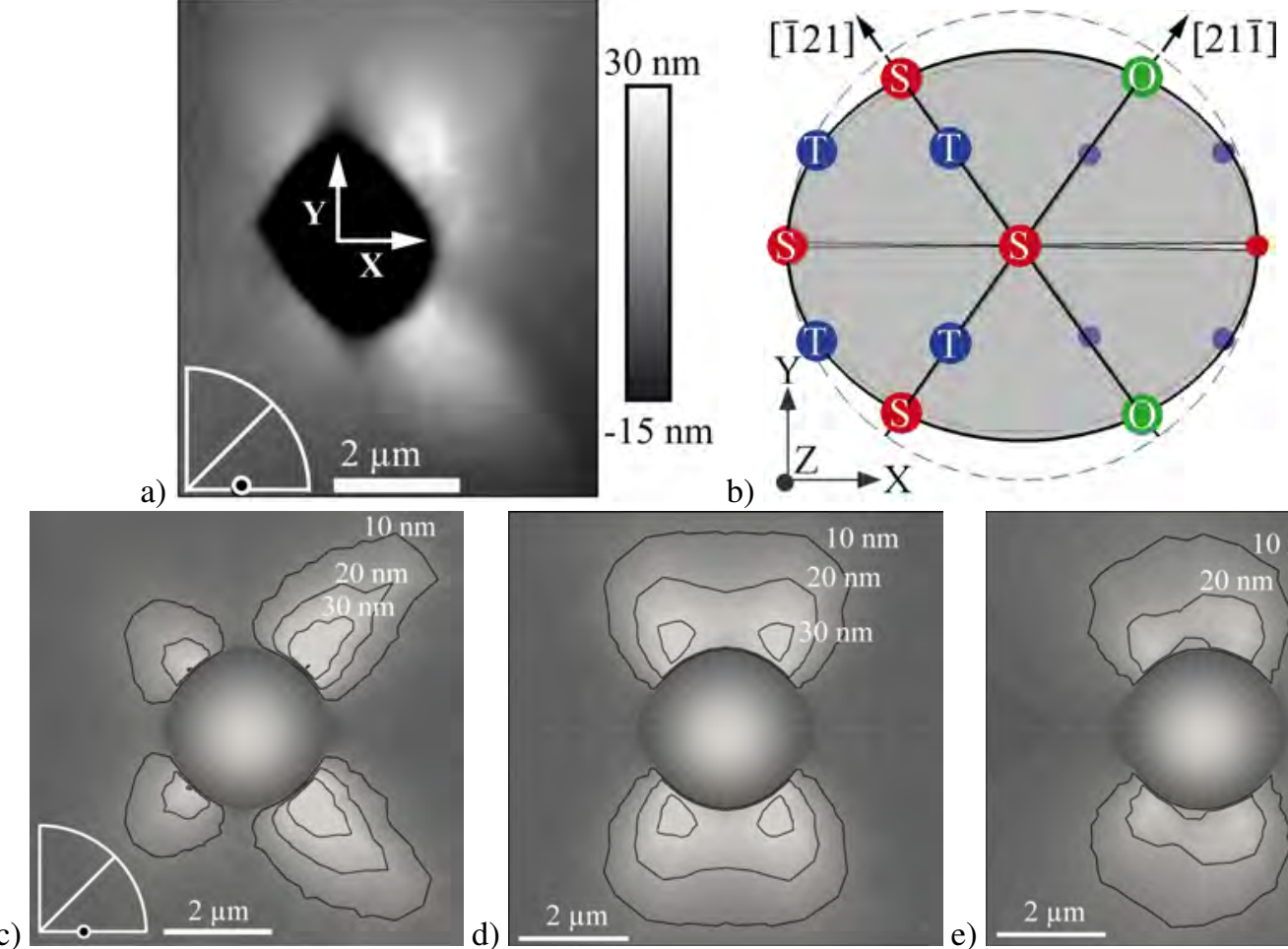

d)

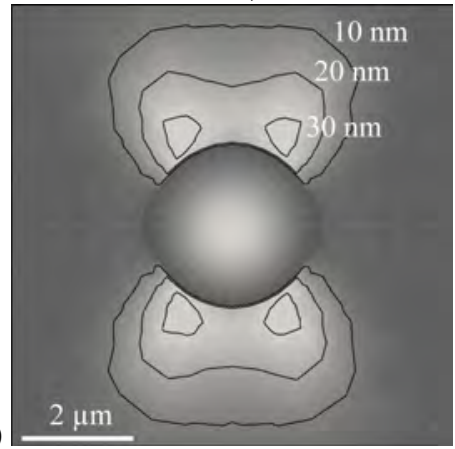

e)

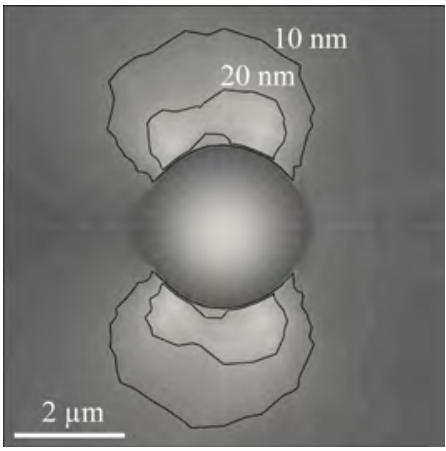

Figure 10: Pile-up topographies for: a) indentation along [13 1111$]$, about $5^{\circ}$ from [ $\left.\begin{array}{lll}1 & 0 & 1\end{array}\right]$, AFM topography; b) Perspective projection of the $\left\{\begin{array}{lll}1 & 1 & 1\end{array}\right\}$-planes and shear directions for exact $\left[\begin{array}{lll}1 & 0 & 1\end{array}\right]$ indentation; green depicts ordinary dislocation glide. c) Simulated indentation along [lll $\left.\begin{array}{ll}0 & 1\end{array}\right], \tau_{c}^{O} / \tau_{c}^{S}<1$ (stoichiometric $\gamma$-TiAl); sphero-conical tip, radius $2.7 \mu \mathrm{m}$, cone-angle $146^{\circ}$; d) A generic fcc-equivalent with $\tau_{c}^{O} / \tau_{c}^{S}=1$; e) Simulated pile-up topography for $\tau_{c}^{O} / \tau_{c}^{S}>1$, expected for Al-rich TiAl. The topographies are displayed after the convention defined in figure 7. 
to the known fcc pile-up patterns, the effect of the strong plastic anisotropy of $\gamma$-TiAl becomes evident. For [ $\left.\begin{array}{lll}1 & 1 & 1\end{array}\right]$-indentation one dominant pile-up hillock was simulated while in fcc crystals the [lll 11 1] pile-up shape exhibits three-fold symmetry $[31,46]$. In fcc crystals the $\left[\begin{array}{lll}1 & 0 & 1\end{array}\right]$ and $\left[\begin{array}{lll}1 & 1 & 0\end{array}\right]$ indentation axes result in equivalent topographies, while in $\gamma$-TiAl the axes result in very different pile-up shapes, figure 8 .

In $\gamma$-TiAl, the $\left[\begin{array}{lll}1 & 0 & 1\end{array}\right]$ indentation axis is particularly suited to investigate the relative strengths of ordinary dislocation glide, $\tau_{c}^{O}$, and superdislocation glide, $\tau_{c}^{S}$. Figure 10 compares the resulting pile-up topographies for the cases $\tau_{c}^{O} / \tau_{c}^{S}<1, \tau_{c}^{O} / \tau_{c}^{S} \approx 1$ and $\tau_{c}^{O} / \tau_{c}^{S}>1$. In the first case, which was also observed experimentally, pronounced pile-up is formed in the $\left[\begin{array}{lll}2 & 1 & \overline{1}\end{array}\right]$ and the $\left[\begin{array}{lll}1 & \overline{1} & \overline{1}\end{array}\right]$ directions, i. e. on the right hand side of the indent. For slip of identical dislocations as in fcc metals, four hillocks of equal height are formed [46]. Easy activation of superdislocation glide systems, as expected for Al-rich $\gamma$-TiAl, leads to pile-up formation similar to the one observed in fcc crystals. Depending on the chosen hardening parameters, the dominant hillocks have also been simulated to appear on the opposite sides compared to stoichiometric $\gamma$-TiAl, in the directions [ $\left[\begin{array}{lll}\overline{2} & \overline{1} & 1\end{array}\right]$ and [ [ $\left.\begin{array}{lll}1 & 2 & 1\end{array}\right]$, i. e. on the left hand side of the indent when displayed according to the in-plane orientation convention. Taking into account these additional simulations, it can be concluded that in $\gamma$-TiAl the $\left[\begin{array}{lll}1 & 0 & 1\end{array}\right]$ indentation direction could be employed as a sensitive indicator of the relative amount of dislocation glide activity on ordinary and superdislocation systems: depending on which glide mechanism is easier to operate, pile-up can potentially form on either side of the indent. 


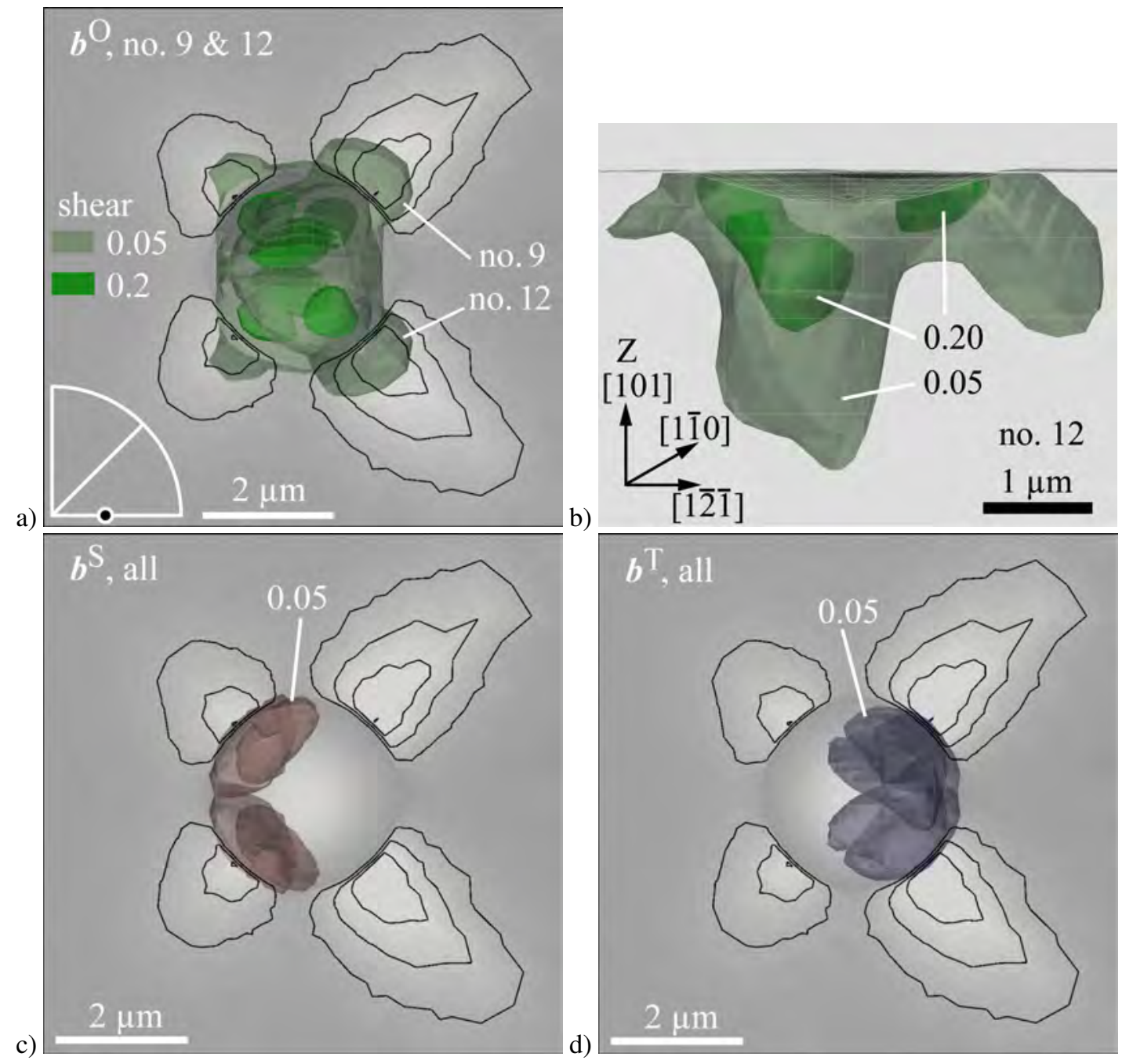

Figure 11: Simulated surface topography from figure 10-c, overlaid with the plastic shear distribution: a) highly activated ordinary slip systems no. 9 \& no. 12 , cf. table 1 , shown by semi-transparent isolevel-surfaces at levels 0.05 and 0.2 , differentiated by saturation; b) shear on system no. 12 , viewing axis perpendicular to the shear direction $\left[\begin{array}{lll}1 & 0\end{array}\right]$; c) all superdislocation systems; red iso-surfaces: shear level 0.05 ; d) all twinning systems; blue iso-surfaces: shear level 0.05 .

The formation of the simulated and experimentally observed pile-up shape in $\left[\begin{array}{lll}1 & 0 & 1\end{array}\right]$ indentation can be explained by analysing the CP-FEM results. Figure 11 relates the crystallographic shear on the individual deformation systems to the upheaval of material as shown by the contour lines. The study of slip activities below the indent confirmed the intuition from the crystallographic relations, figure 10-b, namely, that easy slip on two ordinary dislocation systems is responsible for the two dominating pile-up hillocks. The formation of the pile-up hillocks could be related to the existence of distinct zones of slip activity below the surface, revealed by the simulation. Quantification of the relative magnitude of the slip resistances for ordinary and superdislocations is not straightforward due to the nonlinear influence of cross-hardening; however, the performed simulations suggest that superdislocations can resist shear stresses which are higher by a factor of two to three in comparison to the stresses that will initiate ordinary dislocation glide.

Generally, the orientation dependent pile-up formation corresponds to a projection of the 3D anisotropic plasticity of the material into a 2D height distribution around the indent. In analogy to the measurement of crystallographic texture, i. e. the orientation distribution function (ODF), the resulting picture is not complete by measuring just one pile-up topography or pole figure, respectively. Instead, measurements of more than one indentation axis have to be performed in order to to compile a 3D understanding of the plastic anisotropy. However, for the case of indentation pile-up, the mathematical tools for what is called the pole figure inversion in the realm of texture, still have to be developed. The applicability of non-linear optimization to a similar, computationally less demanding 
inverse problem has been performed recently for Hill's anisotropic elasto-plastic material formulation [97].

Characterization of the orientation dependent pile-up behavior by the presented experimental and computational methods introduces a number of analysis tools: first, the pile-up IPF, figure 8, can be used as a look-up table for a coarse identification of the crystallographic orientation of the indented material. For example, if an fcc structure was used for fast orientation mapping of the $\gamma$-phase by EBSD, in many cases the order variant can be uniquely identified based on the pile-up shape. The determination of crystallographic orientation might be done more exactly by including in the analysis the position of the slip traces [98]. Second, the relative contributions of different deformation systems to the plastic deformation can be characterized by the presented method. The uniqueness of this feature should be stressed. While detailed TEM studies are indispensable for the analysis of the types and interaction of dislocations, statistically significant data is difficult to obtain by TEM alone. This limitation can be overcome by the presented method because a large number of dislocations is active to form the pile-up hillocks.

\subsection{Analysis of active deformation mechanisms}

From the combined investigation by EBSD, AFM and crystal plasticity simulation, an assessment of the deformation mechanism could be performed. The main deformation mechanism for the $\gamma$-phase in a Ti- $46 \mathrm{Al}-8 \mathrm{Nb}$ (at.\%) alloy was ordinary dislocation glide. This appears to be in contrast to earlier findings by Mahapatra et al. [61], who reported twinning as the primary deformation mode. However, in those previous experiments the orientation of the near-stoichiometric layer was such that the Schmid factors for ordinary dislocation glide became minimal. Assuming a high Peierls barrier for superdislocation glide, as also supported by the present work, twinning was left as the only deformation mode providing deformation along the $\left[\begin{array}{lll}0 & 0 & 1\end{array}\right]$ compression axis. Therefore, it is probable that twinning is the predominant deformation mechanism during uniaxial compression of stoichiometric $\gamma$-TiAl along [ $\left[\begin{array}{lll}0 & 0 & 1\end{array}\right]$. However, for more general deformation, mainly ordinary dislocation glide is expected to be responsible for the plastic shape change. This finding is in agreement with conclusions from TEM studies [99].

Regarding the activity of twinning in the analyzed TiAl-Nb alloy it should be mentioned that the nucleation of twins in $\gamma / \alpha_{2}$-microstructures is thought to mainly take place at stress concentrations at the interfaces [57, $100,101]$. Since the aim of the present study was to identify the single phase behavior of $\gamma$-TiAl, unaffected by interfaces, only indentations away from the interfaces have been analyzed. Therefore, a discrepancy between the relative contributions of dislocation glide and twinning is possible for single phase indentation as opposed to macroscopic deformation of two-phase microstructures. At the same time, absence of pronounced twinning in indentation experiments, combined with experimental evidence for high twin activity in microstructures with densely spaced interfaces, can provide an indirect validation of interface nucleation of twins.

\subsection{Error estimation}

The presented method has an advantage that distinguishes it from conventional nanoindentation experiments. It relies only on data that can be measured with a high level of accuracy. These are the indenter load, the residual indent depth and the surface profile. Current resolutions in load transducers are on the order of $1 \mathrm{nN}$ and the displacement resolution is about $0.04 \mathrm{~nm}$ [102]. The surface profiles can be measured by AFM with vertical resolutions much better than $1 \mathrm{~nm}$. In contrast to the measurement of hardness or indentation modulus, which rely on estimated contact areas, the measurement of remaining indent topographies is a robust characterization method that can be carried out with high precision.

Inaccuracies in the presented method could result from inadequate surface preparation. Another source of inaccuracy are possible deviations of the indenter geometry from the ideal shape. Especially deviations from axisymmetry might introduce changes in the pile-up patterns, as can be observed in the extreme case of the Berkovich geometry.

\subsection{Influence of indenter shape on the reproducibility of singl crystal measurements}

Through the use of different indenter geometries, another observation was made which has implications for the use of non-axisymmetric indenters on crystalline samples. As shown in figure 5, the Berkovich indentations lead to different pile-up characteristics when compared to the indents from the sphero-conical geometry. The approximate rule that can be derived from that difference, is that pile-up formation for three-sided pyramidal indenters will only take place if the indenter-face is oriented favorably for the formation of this pile-up. If sharp edges are pointing towards the position of the pile-up from axisymmetric indentation, the formation of this hillock will be reduced or inhibited completely. 
In the cases where geometrically self-similar deformation or localized incipient plasticity is not of high importance, axisymmetric indenters should always be used for indentation testing of single crystals. The influence of pile-up or sink-in on the orientation dependent properties is then identical for identical indentation axes. This does not hold for three-sided pyramidal indenter shapes.

Axisymmetric indenters are particularly suited for the approach developed here. The interaction of nonaxisymmetric geometries will always narrow down the generality of the observed pile-up. Alternatively, the edges of non-axisymmetric indenters would have to be oriented with respect to the crystallographic orientation that is indented. Since this requires significantly increased experimental effort and also the sensitivity of the pile-up formation against even minor misalignments was found to be high, this alternative method is not recommended.

\subsection{Preliminary consideration of hardness anisotropy}

The values from table 3 can be used for an analysis of the hardness anisotropy of $\gamma$-TiAl, not taking into account the orientation dependent changes in contact area through piling-up. The numbers are indicative for an intermediate hardness level of stoichiometric $\gamma$-TiAl for [ $\left[\begin{array}{lll}1 & 0 & 1\end{array}\right]$ indentation. Lower hardness is expected for orientations closer to [llll $\left.\begin{array}{lll}0 & 1\end{array}\right]$ and higher hardness for orientations closer to [ $\left[\begin{array}{lll}1 & 0 & 0\end{array}\right]$-indentation. These preliminary results are also supported by the CP-FEM simulations which additionally predict a global hardness maximum of $\gamma$-TiAl between the $\left[\begin{array}{lll}1 & 1 & 1\end{array}\right]$ axis and the $\left[\begin{array}{lll}1 & 1 & 0\end{array}\right]$ indentation axis. However, the present amount of experimental data is not sufficient to draw an inverse pole figure projection of the anisotropic hardness of $\gamma$-TiAl. The orientation dependence of hardness is much lower compared to the pile-up topographies. The simultaneous action of multiple stress states during indentation makes the hardness also a less anisotropic property than, for example, the uniaxial flow stress. Further, simulations with deactivated cross-hardening revealed that the contribution to the indentation load from the cross-hardening is on the same order of magnitude as the indentation resistance from the selfhardening. Correspondingly, the cross-hardening parameters will have a significant influence on the simulated hardness anisotropy and have to be investigated further.

\section{Conclusions}

The presented nanoindentation analysis combines experimental and computational methods to form a coherent picture of the single phase mechanics of $\gamma$-TiAl. For stoichiometric $\gamma$-TiAl with low interstitial concentrations, as discussed in section 1.2, easy activation of ordinary dislocation glide at room temperature was found to be an intrinsic property, i. e. not mainly caused by dislocations emitted from, or interacting with the interfaces.

The piling-up of material during axisymmetric indentation, is a highly characteristic feature of the prevalent crystallographic deformation processes. Corresponding crystal plasticity finite element simulation is able to analyze and predict the formation of the orientation dependent indent topography.

We showed that sharp axisymmetric tips are better suited for nanoindentation studies of crystallites than nonaxisymmetric indenter shapes. They do not introduce additional geometrical unknowns into the experiment which will lead to scatter in the contact areas and related measures.

A convention has been defined for a systematic presentation of orientation dependence of pile-up topographies, for which the crystallographic orientation of the material is known. The convention can be applied in investigations on any crystal structure indented with an arbitrary indenter geometry. A graphical representation of the orientation dependence of pile-up patterns was introduced. It combines a set of orientations, distributed throughout the standard unit triangle, with the convention for in-plane orientation of the pile-up patterns, to construct the pile-up inverse pole figure.

In summary, a method has been developed to assess the activation of competing deformation mechanisms. Our approach is complementary to the established TEM techniques for the analysis of dislocation-mediated plasticity. The technique has some unique characteristics such as providing reliable statistical information on the operating types of dislocations.

\section{Acknowledgements}

Valuable discussions with Dr. F. Roters (MPI für Eisenforschung) are appreciated. Dr. G. Behr, Dr. W. Löser (both IFW Dresden) and Dr. U. Hecht (ACCESS e.V. Aachen) kindly have provided sample material. Large parts of this work were carried out in the EU FP6 integrated project IMPRESS (NMP3-CT-2004-500635) and this support is gratefully acknowledged. 
Table A.4: Near-equidistant orientations for $\Delta=9^{\circ}$; the 21 orientations cover the fcc unit orientation triangle; full orientations, given as Bunge Euler angles $\left(\varphi_{1}, \Phi, \varphi_{2}\right)$, were defined after the convention given in section $4.2 ;[u v w]$ indices of the indentation axes were calculated with an angular tolerance of $1^{\circ}$ and a $c / a$ ratio of 1.02 ; the spherical coordinates $(\zeta, \eta)$ are defined in section 4.2;

\begin{tabular}{|c|c|c|c|}
\hline № & Bunge Euler angles & {$\left[\begin{array}{lll}u & v & w\end{array}\right]$} & $(\zeta, \eta)$ \\
\hline 1 & $\left(0.0^{\circ}, 0.0^{\circ}, 0.0^{\circ}\right)$ & {$\left[\begin{array}{lll}0 & 0 & 1\end{array}\right]$} & $\left(0.00^{\circ}, 0.00^{\circ}\right)$ \\
\hline 2 & $\left(270.0^{\circ}, 9.0^{\circ}, 90.0^{\circ}\right)$ & {$\left[\begin{array}{lll}1 & 0 & 6\end{array}\right]$} & $\left(0.00^{\circ}, 9.00^{\circ}\right)$ \\
\hline 3 & $\left(315.0^{\circ}, 12.6^{\circ}, 45.0^{\circ}\right)$ & {$\left[\begin{array}{lll}1 & 1 & 6\end{array}\right]$} & $\left(45.00^{\circ}, 12.63^{\circ}\right)$ \\
\hline 4 & $\left(270.0^{\circ}, 18.0^{\circ}, 90.0^{\circ}\right)$ & $\left.\begin{array}{lll}1 & 0 & 3\end{array}\right]$ & $\left(0.00^{\circ}, 18.00^{\circ}\right)$ \\
\hline 5 & $\left(296.0^{\circ}, 19.9^{\circ}, 64.0^{\circ}\right)$ & {$\left[\begin{array}{lll}2 & 1 & 6\end{array}\right]$} & $\left(25.99^{\circ}, 19.87^{\circ}\right)$ \\
\hline 6 & $\left(315.0^{\circ}, 24.7^{\circ}, 45.0^{\circ}\right)$ & $\left.\begin{array}{lll}1 & 1 & 3\end{array}\right]$ & $\left(45.00^{\circ}, 24.68^{\circ}\right)$ \\
\hline 7 & $\left(270.0^{\circ}, 27.0^{\circ}, 90.0^{\circ}\right)$ & $02]$ & $\left(0.00^{\circ}, 27.00^{\circ}\right)$ \\
\hline 8 & $\left(287.3^{\circ}, 28.1^{\circ}, 72.7^{\circ}\right)$ & $16]$ & $\left(17.27^{\circ}, 28.08^{\circ}\right)$ \\
\hline 9 & $\left(302.5^{\circ}, 31.1^{\circ}, 57.5^{\circ}\right)$ & {$\left[\begin{array}{lll}3 & 2 & 6\end{array}\right]$} & $\left(32.53^{\circ}, 31.14^{\circ}\right)$ \\
\hline 10 & $\left(315.0^{\circ}, 35.8^{\circ}, 45.0^{\circ}\right)$ & {$\left[\begin{array}{lll}7 & 7 & 13\end{array}\right]$} & $\left(45.00^{\circ}, 35.78^{\circ}\right)$ \\
\hline 11 & $\left(270.0^{\circ}, 36.0^{\circ}, 90.0^{\circ}\right)$ & {$\left[\begin{array}{lll}3 & 0 & 4\end{array}\right]$} & $\left(0.00^{\circ}, 36.00^{\circ}\right)$ \\
\hline 12 & $\left(282.3^{\circ}, 36.6^{\circ}, 77.7^{\circ}\right)$ & {$\left[\begin{array}{lll}9 & 2 & 12\end{array}\right]$} & $\left(12.30^{\circ}, 36.63^{\circ}\right)$ \\
\hline 13 & $\left(294.1^{\circ}, 38.5^{\circ}, 65.9^{\circ}\right)$ & {$\left[\begin{array}{lll}9 & 4 & 12\end{array}\right]$} & $\left(24.09^{\circ}, 38.52^{\circ}\right)$ \\
\hline 14 & $\left(305.0^{\circ}, 41.6^{\circ}, 55.0^{\circ}\right)$ & {$\left[\begin{array}{lll}3 & 2 & 4\end{array}\right]$} & $\left(35.04^{\circ}, 41.59^{\circ}\right)$ \\
\hline 15 & $\left(315.0^{\circ}, 45.8^{\circ}, 45.0^{\circ}\right)$ & {$\left[\begin{array}{lll}3 & 3 & 4\end{array}\right]$} & $\left(45.00^{\circ}, 45.78^{\circ}\right)$ \\
\hline 16 & $\left(270.0^{\circ}, 45.0^{\circ}, 90.0^{\circ}\right)$ & {$\left[\begin{array}{lll}1 & 0 & 1\end{array}\right]$} & $\left(0.00^{\circ}, 45.00^{\circ}\right)$ \\
\hline 17 & $\left(279.0^{\circ}, 45.4^{\circ}, 81.0^{\circ}\right)$ & {$\left[\begin{array}{lll}6 & 1 & 6\end{array}\right]$} & $\left(9.00^{\circ}, 45.35^{\circ}\right)$ \\
\hline 18 & $\left(288.0^{\circ}, 46.4^{\circ}, 72.0^{\circ}\right)$ & {$\left[\begin{array}{lll}3 & 1 & 3\end{array}\right]$} & $\left(18.00^{\circ}, 46.44^{\circ}\right)$ \\
\hline 19 & $\left(297.0^{\circ}, 48.3^{\circ}, 63.0^{\circ}\right)$ & {$\left[\begin{array}{lll}2 & 1 & 2\end{array}\right]$} & $\left(27.00^{\circ}, 48.30^{\circ}\right)$ \\
\hline 20 & $\left(306.0^{\circ}, 51.0^{\circ}, 54.0^{\circ}\right)$ & {$\left[\begin{array}{lll}4 & 3 & 4\end{array}\right]$} & $\left(36.00^{\circ}, 51.03^{\circ}\right)$ \\
\hline 21 & $\left(315.0^{\circ}, 54.7^{\circ}, 45.0^{\circ}\right)$ & {$\left[\begin{array}{lll}1 & 1 & 1\end{array}\right]$} & $\left(45.00^{\circ}, 54.74^{\circ}\right)$ \\
\hline
\end{tabular}

\section{Appendix A. Generation of near-equidistant orientations}

The near-equidistant directions used for the simulation of nanoindentation were generated after the method presented in the first part of the work by Helming et al. [92]: from the resolution constant, $\Delta$, the near-equidistant directions, $Z_{i j}$, are calculated by

$$
Z_{i j}=\left(\begin{array}{c}
x \\
y \\
z
\end{array}\right)=\frac{1}{N_{i j}}\left(\begin{array}{c}
1 \\
\tan (i \Delta) \\
\tan (j \Delta)
\end{array}\right), \text { with } N_{i j}=\sqrt{1+\tan ^{2}(i \Delta)+\tan ^{2}(j \Delta)}, \quad-\tau<i, j \leq \tau
$$

and integer indices, $i, j$, and integer $\tau=90^{\circ} / \Delta$.

For a resolution constant, $\Delta$, of $9^{\circ}$, the method results in 21 directions throughout the cubic standard orientation triangle [ $\left[\begin{array}{lll}0 & 0 & 1\end{array}\right]-\left[\begin{array}{lll}1 & 1 & 0\end{array}\right]-\left[\begin{array}{lll}1 & 1 & 1\end{array}\right]$, table A.4. Angular distances between these directions vary from about $7.3^{\circ}$ to $9^{\circ}$. The full orientations follow the system described in section 4.2. The used Euler angles follow the Bunge description and define three successive rotations about $Z, X^{\prime}$, and $Z^{\prime}$ that will rotate the indenter coordinate system into the crystal coordinate system [103].

The 21 orientations can be transformed to 63 orientations in the unit triangle [ $\left[\begin{array}{lll}0 & 0 & 1\end{array}\right]-\left[\begin{array}{lll}1 & 0 & 0\end{array}\right]-\left[\begin{array}{llll}1 & 1 & 0\end{array}\right]$ of the tetragonal structure, of which 51 orientations are unique. If improper rotations are not allowed, the larger unit triangle [ll $\left.\begin{array}{lll}0 & 0 & 1\end{array}\right]-\left[\begin{array}{lll}1 & 0 & 0\end{array}\right]-\left[\begin{array}{lll}0 & 1 & 0\end{array}\right]$ has to be used, resulting in 91 orientations. Coarser (e.g. $\left.\Delta=15^{\circ}\right)$ or finer (e.g. $\Delta=5^{\circ}$ ) discretizations of the orientation space can be generated.

\section{References}

[1] D. Shechtman, M. J. Blackburn, H. Lipsitt, The plastic deformation of TiAl, Metall. Trans. 5 (1974) 1373-1381.

[2] Y. W. Kim, Ordered Intermetallic Alloys. 3. Gamma-Titanium Aluminides, JOM 46 (7) (1994) 30-39.

[3] F. Appel, R. Wagner, Microstructure and deformation of two-phase gamma-titanium aluminides, Mater. Sci. Engr. R22 (1998) 187-268, doi:10.1016/S0927-796X(97)00018-1.

[4] Y. W. Kim, Strength and ductility in TiAl alloys, Intermetallics 6 (7) (1998) 623-628.

[5] H. Kestler, H. Clemens, Production, Processing and Application of $\gamma$ (TiAl)-Based Alloys, in: C. Leyens, M. Peters (Eds.), Titanium and Titanium Alloys: Fundamentals and Applications, chap. 14, Wiley-VCH, ISBN 3527305343, 351-392, 2003.

[6] H. Mecking, C. Hartig, U. F. Kocks, Deformation modes in gamma-TiAl as derived from the single crystal yield surface, Acta Mater. 44 (4) (1996) 1309-1321.

[7] V. K. Vasudevan, M. A. Stucke, S. A. Court, H. L. Fraser, The Influence of 2nd Phase $\mathrm{Ti}_{3} \mathrm{Al}$ on the Deformation Mechanisms in TiAl, Philos. Mag. Lett. 59 (6) (1989) 299-307.

[8] H. Inui, M. Oh, A. Nakamura, Room-temperature tensile deformation of polysynthetically twinned (PST) crystals of TiAl, Acta Metall. Mater. 40 (11) (1992) 3095-3104.

[9] M. A. Morris, Dislocation configurations in two phase TiAl alloys. I. Annealed and indented structures, Philos. Mag. A 68 (2) (1993) 237-257, doi:10.1080/01418619308221203.

[10] K. Kishida, H. Inui, M. Yamaguchi, Deformation of lamellar structure in TiAl-Ti 3 Al two phase alloys, Philos. Mag. A 78 (1998) 1-28. 
[11] F. Appel, U. Christoph, Coherency stresses and interface-related deformation phenomena in two-phase titanium aluminides, Intermetallics 7 (1999) 1173-1182.

[12] M. A. Gibson, C. T. Forwood, Slip transfer of deformation twins in duplex gamma-based Ti-Al alloys. Part I. Transfer across gammagamma coherent twin interfaces, Philos. Mag. A 80 (12) (2000) 2747-2783.

[13] C. T. Forwood, M. A. Gibson, Slip transfer of deformation twins in duplex gamma-based Ti-Al alloys. Part II. Transfer across gammaalpha(2) interfaces, Philos. Mag. A 80 (12) (2000) 2785-2811.

[14] M. A. Gibson, C. T. Forwood, Slip transfer of deformation twins in duplex gamma-based Ti-Al alloys - Part III. Transfer across general large-angle gamma-gamma grain boundaries, Philos. Mag. A 82 (7) (2002) 1381-1404.

[15] A. Couret, H. A. Calderon, P. Veyssiere, Intralamellar dislocation networks formed by glide in gamma-TiAl - I. The mechanism of formation, Philos. Mag. 83 (14) (2003) 1699-1718, doi:10.1080/1478643031000081707.

[16] D. Tabor, The hardness of metals, Clarendon Press, Oxford University Press, Oxford, London, UK, 1951.

[17] N. Gane, F. P. Bowden, Microdeformation of Solids, J. Appl. Phys. 39 (3) (1968) 1432-1435.

[18] N. Gane, Direct Measurement of Strength of Metals on a Sub-micrometre Scale, Proc. Roy. Soc. London A 317 (1530) (1970) $367-391$.

[19] N. Gane, J. M. Cox, Micro-hardness of Metals at Very Low Loads, Philos. Mag. 22 (179) (1970) 881-891, doi: 10.1080/14786437008221059.

[20] S. I. Bulychev, V. P. Alekhin, M. K. Shorshorov, A. P. Ternovskii, G. D. Shnyrev, Determination of Youngs Modulus According to Indentation Diagram, Zavodskaya Laboratoriya 41 (9) (1975) 1137-1140.

[21] F. Fröhlich, P. Grau, W. Grellmann, Performance and analysis of recording microhardness tests, Phys. Status Solidi A 42 (1) (1977) 79-89, doi:10.1002/pssa.2210420106.

[22] J. B. Pethica, R. Hutchings, W. C. Oliver, Hardness Measurement at Penetration Depths as Small as 20-nm, Philos. Mag. A 48 (4) (1983) 593-606.

[23] M. Doerner, W. Nix, A method for interpreting the data from depth-sensing indentation instruments, J. Mater. Res. 1 (4) (1986) 601-609, doi:10.1557/JMR.1986.0601.

[24] A. Fischer-Cripps, Nanoindentation, Springer, 2nd edn., 2004.

[25] K. J. Van Vliet, J. Li, T. Zhu, S. Yip, S. Suresh, Quantifying the early stages of plasticity through nanoscale experiments and simulations, Phys. Rev. B 67 (10) (2003) 104105-1-15, doi:10.1103/PhysRevB.67.104105.

[26] C. A. Schuh, Nanoindentation studies of materials, Materials today 9 (5) (2006) 32-40, doi:10.1016/S1369-7021(06)71495-X.

[27] E. Carrasco, O. R. D. L. Fuente, J. Rojo, Dislocation emission at the onset of plasticity during nanoindentation in gold, Philos. Mag. 88 (3) (2008) 281-296, doi:10.1080/14786430701798951.

[28] C. Brookes, J. O’Neill, B. Redfern, Anisotropy in Hardness of Single Crystals, Proc. Roy. Soc. London A 322 (1548) (1971) $73-88$.

[29] G. Tammann, A. Müller, Über Verfahren zur Bestimmung der Orientierung der Kristallite, Z. Metallkd. 18 (1926) 69-79.

[30] F. Osmond, G. Cartaud, Figures of pressure or percussion on crystallised plastic metals, Comptes Rendus Hebdomadaires Des Seances De L Academie Des Sciences 141 (1905) 122-124.

[31] F. Osmond, G. Cartaud, Die Kristallographie des Eisens, Metallurgie - Zeitschrift für die gesamte Hüttenkunde 15 (1906) $522-545$.

[32] R. Smith, D. Christopher, S. D. Kenny, A. Richter, B. Wolf, Defect generation and pileup of atoms during nanoindentation of Fe single crystals, Phys. Rev. B 67 (24), doi:10.1103/PhysRevB.67.245405.

[33] M. Biener, J. Biener, A. Hodge, A. Hamza, Dislocation nucleation in bcc Ta single crystals studied by nanoindentation, Phys. Rev. B 76 (16) (2007) 165422-1-6, doi:10.1103/PhysRevB.76.165422.

[34] Y. H. Lee, J. H. Hahn, S. H. Nahm, J. I. Jang, D. Kwon, Investigations on indentation size effects using a pile-up corrected hardness, J. Phys. D: Appl. Phys. 41 (7) (2008) 074027 (5pp), doi:10.1088/0022-3727/41/7/074027.

[35] A. Fischer-Cripps, Critical review of analysis and interpretation of nanoindentation test data, Surf. Coat. Technol. 200 (14-15) (2006) 4153-4165, doi:10.1016/j.surfcoat.2005.03.018.

[36] A. K. Bhattacharya, W. D. Nix, Finite-element Simulation of Indentation Experiments, Int. J. Solids Structures 24 (9) (1988) 881-891.

[37] R. Hill, F. Storakers, A. Zdunek, A theroretical study of the Brinnell hardness test, Proc. Roy. Soc. Lond. A 423 (1989) $301-330$.

[38] T. A. Laursen, J. C. Simo, A Study of the Mechanics of Microindentation Using Finite-Elements, J. Mater. Res. 7 (3) (1992) 618-626, doi:10.1557/JMR.1992.0618.

[39] A. E. Giannakopoulos, P. L. Larsson, R. Vestergaard, Analysis of Vickers Indentation, Int. J. Solids Structures 31 (19) (1994) $2679-2708$.

[40] A. Bolshakov, G. M. Pharr, Influences of pileup on the measurement of mechanical properties by load and depth sensing indentation techniques, J. Mater. Res. 13 (4) (1998) 1049-1058, doi:10.1557/JMR.1998.0146.

[41] J. A. Knapp, D. M. Follstaedt, S. M. Myers, J. C. Barbour, T. A. Friedmann, Finite-element modeling of nanoindentation, J. Appl. Phys. 85 (3) (1999) 1460-1474, doi:10.1063/1.369178.

[42] S. D. Mesarovic, N. A. Fleck, Spherical indentation of elastic-plastic solids, Proc. Roy. Soc. London A 455 (1987) (1999) $2707-2728$.

[43] M. Dao, N. Chollacoop, K. J. V. Vliet, T. A. Venkatesh, S. Suresh, Computational modeling of the forward and reverse problems in instrumented sharp indentation, Acta Mater. 49 (19) (2001) 3899-3918, doi:10.1016/S1359-6454(01)00295-6.

[44] K. Durst, B. Backes, O. Franke, M. Göken, Indentation size effect in metallic materials: Modeling strength from pop-in to macroscopic hardness using geometrically necessary dislocations, Acta Mater. 54 (9) (2006) 2547-2555, doi:10.1016/j.actamat.2006.01.036.

[45] P. L. Larsson, Modelling of sharp indentation experiments: some fundamental issues, Philos. Mag. 86 (33-35) (2006) 5155-5177, doi:10.1080/14786430600589089.

[46] Y. Wang, D. Raabe, C. Klüber, F. Roters, Orientation dependence of nanoindentation pile-up patterns and of nanoindentation microtextures in copper single crystals, Acta Mater. 52 (8) (2004) 2229-2238, doi:10.1016/j.actamat.2004.01.016.

[47] N. Zaafarani, D. Raabe, R. N. Singh, F. Roters, S. Zaefferer, Three-dimensional investigation of the texture and microstructure below a nanoindent in a Cu single crystal using 3D EBSD and crystal plasticity finite element simulations, Acta Mater. 54 (7) (2006) 1863-1876, doi:10.1016/j.actamat.2005.12.014. 
[48] N. Zaafarani, D. Raabe, F. Roters, S. Zaefferer, On the origin of deformation-induced rotation patterns below nanoindents, Acta Mater. 56 (1) (2008) 31-42, doi:10.1016/j.actamat.2007.09.001.

[49] Y. Liu, B. Wang, M. Yoshino, S. Roy, H. Lu, R. Komanduri, Combined numerical simulation and nanoindentation for determining mechanical properties of single crystal copper at mesoscale, J. Mech. Phys. Solids 53 (2005) 2718-2741.

[50] Y. Liu, S. V. J. Ma, M. Y. H. Lu, R. Komanduri, Orientation effects in nanoindentation of single crystal copper, Int. J. Plast. 24 (11) (2008) 1990-2015, doi:10.1016/j.ijplas.2008.02.009.

[51] O. Casals, S. Forest, Finite element crystal plasticity analysis of spherical indentation in bulk single crystals and coatings, Comput. Mater. Sci. 45 (3) (2009) 774-782, doi:10.1016/j.commatsci.2008.09.030.

[52] C. Kearney, Z. Zhao, B. J. F. Bruet, R. Radovitzky, M. C. Boyce, C. Ortiz, Nanoscale anisotropic plastic deformation in single crystal aragonite, Phys. Rev. Lett. 96 (25), doi:10.1103/PhysRevLett.96.255505.

[53] A. Gerday, M. B. Bettaieb, L. Duchêne, N. Clément, H. Diarra, A. Habraken, Interests and limitations of nanoindentation for bulk multiphase material identification: Application to the [beta] phase of Ti-5553, Acta Mater. 57 (2009) 5186-5196, doi: 10.1016/j.actamat.2009.07.020.

[54] C. Zambaldi, F. Roters, D. Raabe, U. Glatzel, Modeling and experiments on the indentation deformation and recrystallization of a single-crystal nickel-base superalloy, Mater. Sci. Engr. A 454 (2007) 433-440, doi:10.1016/j.msea.2006.11.068.

[55] H. Inui, A. Nakamura, M. H. Oh, M. Yamaguchi, Deformation Structures in Ti-rich TiAl Polysynthetically Twinned Crystals, Philos. Mag. A 66 (4) (1992) 557-573.

[56] Y. Liu, D. L. Lin, Z. G. Liu, Z. G. Wang, Superlattice dislocations in Ti-rich polysynthetically twinned crystals of TiAl, Philosophical Magazine A 79 (12) (1999) 2965-2978.

[57] F. Appel, An electron microscope study of mechanical twinning and fracture in TiAl alloys, Philos. Mag. 85 (2) (2005) $205-231$.

[58] B. Skrotzki, Crystallographic aspects of deformation twinning and consequences for plastic deformation processes in $\gamma$-TiAl, Acta Mater. 48 (2000) 851-862, doi:10.1016/S1359-6454(99)00385-7.

[59] A. Denquin, S. Naka, Phase-transformation mechanisms involved in two-phase TiAl-based alloys -I. Lamellar structure formation, Acta Mater. 44 (1996) 343-352.

[60] N. Bird, S. Jiao, G. Taylor, The formation of ordered domains during crystal growth of [gamma]-TiAl, Intermetallics 8 (2) (2000) 133-141, doi:10.1016/S0966-9795(99)00077-1.

[61] R. Mahapatra, A. Girshick, D. P. Pope, V. Vitek, Deformation Mechanisms of Near-Stoichiometric Single-phase TiAl Single-Crystals - a Combined Experimental and Atomistic Modeling Study, Scripta Metall. Mater. 33 (12) (1995) 1921-1927.

[62] E. Hall, S.-C. Huang, Stoichiometry effects: on the deformation of binary TiAl alloys, J. Mater. Res. 4 (3) (1989) 595-602, doi: 10.1557/JMR.1989.0595.

[63] H. Inui, M. Matsumuro, D. H. Wu, M. Yamaguchi, Temperature dependence of yield stress, deformation mode and deformation structure in single crystals of TiAl (Ti-56 at\% Al), Philos. Mag. A 75 (2) (1997) 395-423.

[64] M. Zupan, K. Hemker, Yielding behaviour of aluminum-rich single crystalline gamma-TiAl, Acta Mater. 51 (2003) 6277-6290.

[65] M. Aindow, K. Chaudhuri, S. Das, H. L. Fraser, On the Influence of Stoichiometry and Purity on the Deformation Mechanisms in the Intermetallic Compound TiAl, Scripta Metall. Mater. 24 (6) (1990) 1105-1108.

[66] S. A. Court, V. K. Vasudevan, H. L. Fraser, Deformation Mechanisms in the Intermetallic Compound TiA1, Philos. Mag. A 61 (1) (1990) $141-158$.

[67] B. K. Kad, H. L. Fraser, Effects of Oxygen on the Deformation-Behavior in Single-Phase Gamma-TiAl Alloys, Philos. Mag. Lett. 70 (4) (1994) 211-220.

[68] D. Häussler, M. Bartsch, M. Aindow, I. P. Jones, U. Messerschmidt, Dislocation processes during the plastic deformation of gammaTiAl, Philos. Mag. A 79 (5) (1999) 1045-1071.

[69] R. Uemori, T. Hanamura, H. Morikawa, Oxygen Scavenging Effect of the Alpha-2 Phase in the TiAl IntermetallicCompound, Scripta Metall. Mater. 26 (6) (1992) 969-974.

[70] M. A. Morris, Dislocation mobility, ductility and anomalous strengthening of two-phase TiAl alloys: effects of oxygen and composition, Intermetallics 4 (5) (1996) 417-426.

[71] M. Göken, M. Kempf, W. Nix, Hardness and modulus of the lamellar microstructure in PST-TiAl studied by nanoindentations and AFM, Acta Mater. 49 (5) (2001) 903-911, doi:10.1016/S1359-6454(00)00375-X.

[72] J. Kempf, M. Göken, H. Vehoff, The mechanical properties of different lamellae and domains in PST-TiAl investigated with nanoindentations and atomic force microscopy, Mater. Sci. Engr. A 329 (Sp. Iss. SI) (2002) 184-189, doi:10.1016/S0921-5093(01)01561-1.

[73] M. H. Zhao, W. S. Slaughter, M. Li, S. X. Mao, Material-length-scale-controlled nanoindentation size effects due to strain-gradient plasticity, Acta Mater. 51 (15) (2003) 4461-4469, doi:10.1016/S1359-6465(03)00281-7.

[74] W. D. Nix, H. J. Gao, Indentation size effects in crystalline materials: A law for strain gradient plasticity, J. Mech. Phys. Solids 46 (3) (1998) 411-425.

[75] D. Souptel, W. Löser, G. Behr, Vertical optical floating zone furnace: Principles of irradiation profile formation, J. Cryst. Growth 300 (2) (2007) 538-550, doi:10.1016/j.jcrysgro.2006.12.042.

[76] C. Zambaldi, S. Zaefferer, S. Wright, Characterization of order domains in $\gamma$-TiAl by orientation microscopy based on electron backscatter diffraction, J. Appl. Crystallogr. 42 (6) (2009) 1092-1101, doi:10.1107/S0021889809036498.

[77] F. Roters, Application of crystal plasticity FEM from single crystal to bulk polycrystal, Comput. Mater. Sci. 32 (3-4) (2005) 509-517, doi:10.1016/j.commatsci.2004.09.014.

[78] F. Roters, P. Eisenlohr, L. Hantcherli, D. D. Tjahjanto, T. R. Bieler, D. Raabe, Overview of constitutive laws, kinematics, homogenization, and multiscale methods in crystal plasticity finite element modeling: theory, experiments, applications, Acta Mater. 58 (4) (2010) 1152-1211.

[79] S. R. Kalidindi, C. A. Bronkhorst, L. Anand, Crystallographic Texture Evolution in Bulk Deformation Processing of FCC Metals, J. Mech. Phys. Solids 40 (3) (1992) 537-569, doi:10.1016/0022-5096(92)80003-9.

[80] E. H. Lee, Elastic-plastic Deformation at Finite Strains, J. Appl. Mechanics 36 (1) (1969) 1-6. 
[81] S. Kalidindi, Micro-Mechanical Finite Element Models for Crystal Plasticity, in: D. Raabe, F. Roters, F. Barlat, L.-Q. Chen (Eds.), Continuum Scale Simulation of Engineering Materials, Wiley-VCH, 529-542, 2004.

[82] D. Peirce, R. J. Asaro, A. Needleman, Material Rate Dependence and Localized Deformation in Crystalline Solids, Acta Metall. 31 (12) (1983) 1951-1976, doi:10.1016/0001-6160(83)90014-7.

[83] R. J. Asaro, A. Needleman, Overview 42. Texture Development and Strain-Hardening in Rate Dependent Polycrystals, Acta Metall. 33 (6) (1985) 923-953.

[84] R. Hill, Generalized Constitutive Relations for Incremental Deformation of Metal Crystals by Multislip, J. Mech. Phys. Solids 14 (2) (1966) 95-102, doi:10.1016/0022-5096(66)90040-8

[85] D. Peirce, R. J. Asaro, A. Needleman, An Analysis of Nonuniform and Localized Deformation in Ductile Single-crystals, Acta Metall. 30 (6) (1982) 1087-1119, doi:10.1016/0001-6160(82)90005-0.

[86] S. Kalidindi, Incorporation of deformation twinning in crystal plasticity models, J. Mech. Phys. Solids 46 (2) (1998) 267-290.

[87] M. H. Yoo, Twinning and mechanical behavior of titanium aluminides and other intermetallics, Intermetallics 6 (7) (1998) 597-602, doi:10.1016/S0966-9795(98)00033-8.

[88] MSC Software, Marc2007r1, Volume D — User Subroutines and Special Routines, 2007.

[89] Y. He, R. B. Schwarz, T. Darling, M. Hundley, S. H. Whang, Z. M. Wang, Elastic constants and thermal expansion of single crystal gamma-TiAl from 300 to 750 K, Mater. Sci. Engr. A 240 (1997) 157-163.

[90] B. Yang, H. Vehoff, Dependence of nanohardness upon indentation size and grain size - A local examination of the interaction between dislocations and grain boundaries, Acta Mater. 55 (3) (2007) 849-856.

[91] A. Morawiec, Orientations and Rotations - Computations in Crystallographic Textures, Springer, ISBN 3-540-40734-0, 2004.

[92] K. Helming, R. Tamm, B. Fels, An Automated Component Method, Mater. Sci. Forum 273-275 (1998) 119-124.

[93] T. Parthasarathy, M. G. Mendiratta, D. M. Dimiduk, Flow behavior of PST and fully lamellar polycrystals of Ti-48Al in the microstrain regime, Acta Mater. 46 (11) (1998) 4005-4016.

[94] K. Maruyama, M. Yamaguchi, G. Suzuki, H. Zhu, H. Y. Kim, M. Yoo, Effects of lamellar boundary structural change on lamellar size hardening in TiAl alloy, Acta Mater. 52 (2004) 5185-5194.

[95] B. Kad, P. Hazzledine, Shear boundaries in lamellar TiAl, Philos. Mag. Lett. 66 (3) (1992) 133-139.

[96] P. Hazzledine, Coherency and loss of coherency in TiAl, Intermetallics 6 (1998) 673-677.

[97] M. Bocciarelli, G. Bolzon, G. Maier, Parameter identification in anisotropic elastoplasticity by indentation and imprint mapping, Mech. Mater. 37 (8) (2005) 855-868, doi:10.1016/j.mechmat.2004.09.001.

[98] S. C. Chang, H. C. Chen, The determination of F.C.C. crystal orientation by indentation, Acta Metall. Mater. 43 (6) (1995) 2501-2505, doi:10.1016/0956-7151(94)00420-X

[99] F. Appel, U. B. U. Christoph, S. Eggert, P. Janschek, U. Lorenz, J. Müllauer, M. Oehring, J. Paul, Recent progress in the Development of Gamma Titanium Aluminide Alloys, Adv. Engr. Mater. 2 (11) (2000) 699-720.

[100] F. Appel, P. A. Beaven, R. Wagner, Deformation Processes Related to Interfacial Boundaries in 2-phase Gamma-titanium Aluminides, Acta Metall. Mater. 41 (6) (1993) 1721-1732.

[101] L. Hsiung, T. G. Nieh, B. W. Choi, J. Wadsworth, Interfacial dislocations and deformation twinning in fully lamellar TiAl, Mater. Sci. Engr. A 329-331 (2002) 637-643.

[102] Hysitron Inc., TriboIndenter User's Manual, 2003.

[103] H. Bunge, Texture Analysis in Materials Science, Butterworths Publishers, London, 1982. 